\title{
A New Detection Scheme of Wave-Breaking Events with Blocking Flow Configurations
}

\author{
NING SHI ${ }^{\mathrm{a}}$ AND HISASHI NAKAMURA ${ }^{\mathrm{b}}$

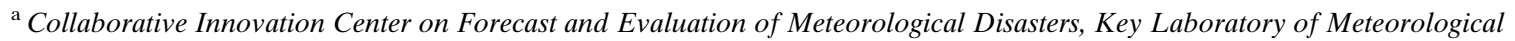 \\ Disaster of Ministry of Education, Nanjing University of Information Science and Technology, Nanjing, China \\ ${ }^{\mathrm{b}}$ Research Center for Advanced Science and Technology, The University of Tokyo, Tokyo, Japan
}

(Manuscript received 21 January 2020, in final form 15 November 2020)

\begin{abstract}
Blocking flow configurations, which tend to accompany strong circulation anomalies and therefore can cause extreme weather conditions, have recently been studied in relation to large-scale wave breaking (WB). Although WB events have been detected often from an instantaneous morphology perspective, the present study proposes a new approach for the detection from a wave-activity perspective in focusing on its accumulation, saturation, and release. This evolution of wave activity is theoretically equivalent to anomalous potential vorticity (PV) flux with its sign changing from negative to positive, which is utilized in this study to detect WB events that accompany high-amplitude height anomalies and blocking flow configurations. As in previous studies, a given WB event is classified into a high pressure type or low pressure type depending upon the sign of the primary PV anomaly center and further into an eastward or westward type depending upon the longitudinal movement of that center. The new method applied to the wintertime Northern Hemisphere shows that a WB event with a blocking anticyclone is likely to accompany an eastward-moving PV anomaly center, occurring mostly under anticyclonic westerly shear. By contrast, a WB event with a strong cyclonic anomaly mostly accompanies the eastward-moving PV anomaly center under cyclonic westerly shear. Composite analysis confirms the consistency between the sign-changing anomalous PV flux and convergence/divergence of wave-activity flux of quasi-stationary Rossby wave trains around the $\mathrm{WB}$ region.
\end{abstract}

KEYWORDS: Blocking; Rossby waves; Synoptic climatology; Wave breaking

\section{Introduction}

The concept of potential vorticity (PV) was first introduced in a barotropic framework by Rossby (1940), and then its ultimate baroclinic form was derived by Ertel (1942). Under adiabatic and frictionless conditions, PV is materially conserved and so is potential temperature, meaning that the movement of PV contours on isentropic surfaces tracks the motion of air masses. PV is thus suited for studying time evolution of the circulation as an excellent dynamical tracer for air masses. Hoskins et al. (1985) provided a full account of PV and its theoretical importance, including the properties of material conservation of PV and invertibility. The invertibility means that all details of a balanced flow can be determined if the PV distribution is known everywhere on isentropic surfaces, subject to suitable boundary conditions and a knowledge of the total mass between isentropic surfaces. Today, the PV perspective is widely used in dynamical studies of mid- and highlatitude weather systems.

In recent years, many studies have been carried out on wave breaking (WB) from a PV perspective. For example, Pelly and Hoskins (2003) demonstrated that WB can be quantitatively measured with an index $(B)$ that represents macroscale meridional gradient of potential temperature around a reference latitude, which corresponds to a storm track axis at a given longitude, on the surface of $2 \mathrm{PV}$ units (PVU) $\left(\theta_{2 \mathrm{PVU}}\right.$ for brevity). If the meridional gradient is reversed $(B>0)$, then WB is considered to occur instantaneously and locally. This method is hereafter referred to as the PV $-\theta$ method. They

Corresponding author: Dr. Ning Shi, shining@nuist.edu.cn further proposed that midlatitude blocking episodes can be understood as persistent occurrence of WB over a certain longitudinal extent. Therefore, the $B$ index can be useful for identifying midlatitude blocking episodes if thresholds are set for both longitudinal extent and persistence of a given local WB (Pelly and Hoskins 2003). To detect high-latitude blockings (Berrisford et al. 2007; Woollings et al. 2008), Masato et al. (2013) modified the PV $-\theta$ method by removing the constraint on the reference latitude to make the $B$ index available over the mid- and high-latitude regions.

Some other studies, however, questioned whether the relationship between local WB and a blocking flow configuration is indeed close. If blocking episodes are detected as prominent anticyclonic anomalies (Dole and Gordon 1983; Schwierz et al. 2004; Nishii et al. 2011), the relationship between local WB and blocking may not necessarily be as close as suggested by the PV- $\theta$ method. In fact, Schwierz et al. (2004) argued that local reversal of gradient of a given variable, such as tropopauselevel potential temperature (equivalent to $\theta_{2 \mathrm{PVU}}$ ) or $500-\mathrm{hPa}$ height, does not necessarily accompany an equivalent-barotropic blocking anticyclone. Altenhoff et al. (2008) pointed out that despite a statistically significant increase in the WB frequency during blocking high episodes compared to the climatological frequency, WB is observed on only $36 \%$ and $42 \%$ of the total days of the Pacific and Atlantic blocking highs, respectively. Small et al. (2014) also confirmed a rather weak relationship between WB and blocking highs over the eastern Pacific. Thus, WB and blocking may not necessarily be regarded as two aspects of the same phenomenon.

In addition, other studies, including Nakamura et al. (1997), Nakamura and Fukamachi (2004), Takaya and Nakamura 
(2005a,b), Nishii et al. (2010), Wolf et al. (2018), and Zschenderlein et al. (2018), demonstrated that some of the blocking flow configurations develop in association with local breaking of quasi-stationary Rossby wave trains. Here, the local breaking means the accumulation and release of wave activity, as indicated by the convergence and divergence of wave-activity flux, which was first pointed out in a blocking study by Nakamura (1994). The impacts of local breaking of quasi-stationary Rossby wave trains on the blocking formation can be of regional and seasonal dependence. For example, the blocking high formation associated with local breaking of quasi-stationary Rossby wave trains is more evident over the continents (e.g., Eurasia) than that over maritime regions (e.g., North Pacific) in boreal winter (Nakamura et al. 1997; Takaya and Nakamura 2005a,b), and more evident in July than May over the Far East (Nakamura and Fukamachi 2004). Therefore, it is worth clarifying the preferred regions for the WB episodes with blocking flow configurations. As revealed by Nakamura (1994), Nakamura et al. (1997) and Nakamura and Fukamachi (2004), the local breaking of a quasi-stationary Rossby wave train is characterized by the negative correlation between anomalies in PV and meridional wind velocity. Motivated by these works, our new WB index is constructed by using the anomalous PV flux, as described in detail in section $2 b(2)$.

Barriopedro et al. (2010) and Dunn-Sigouin et al. (2013) proposed novel blocking indices, which are based on both absolute (or total) and departure (anomaly) fields, by reconciling the two conventional approaches. A detailed comparison between the detection method of blocking based on absolute field and that on departure fields has been performed by Pinheiro et al. (2019). The "absolute-field" detection is used to depict the reversal of the flow direction (Rex 1950; Lejenäs and Økland 1983; Tibaldi and Molteni 1990; Pelly and Hoskins 2003), while the "departure-field" detection method emphasizes extreme anomalies (Dole and Gordon 1983; Schwierz et al. 2004; among others). As reviewed by Barriopedro et al. (2010), so-called departure fields can be defined locally as deviations from the time-averaged fields (i.e., anomalies from the climatological-mean field) or from a spatial mean (e.g., an instantaneous regional mean field). Inspired by Barriopedro et al. (2010) and Dunn-Sigouin et al. (2013), WB events with blocking flow configurations are detected also in both absolute and departure fields in this study.

Morphologically, WB at the dynamical tropopause is classified into anticyclonic and cyclonic orientations under the dominance of high and low pressure anomalies, respectively (Thorncroft et al. 1993; Peters and Waugh 1996; Tyrlis and Hoskins 2008b). Masato et al. (2012) proposed the following two indices to characterize WB features in blocking events. The "direction of breaking" (DB) index is calculated from the zonal gradient of meridionally averaged $\theta_{2 \mathrm{PVU}}$ around the WB center, while the "relative intensity" (RI) index is measured by the difference of meridionally averaged $\theta_{2 \mathrm{PVU}}$ at the WB center from its local climatological mean. The WB center in their study is defined as the grid point at which the reversed meridional gradient of the potential temperature is largest on the tropopause level. The DB index at the onset day can be used for determining whether a given blocking event is an anticyclonic or cyclonic type, in recognition of the tendency for overturning direction to remain the same throughout the event. The RI index is averaged over the whole period to determine whether the event at a given instance is characterized by a blocking high or low. Thus, a combination of the DB and RI indices proposed by Masato et al. (2012) is designed to capture overall characteristics of WB during a given blocking episode. Several other algorithms similar to the DB index have been proposed mainly through identifying some particular key grid points on representative circumpolar contours (Martius et al. 2007; Strong and Magnusdottir 2008; Rivière et al. 2010; Weijenborg et al. 2012). Those algorithms resemble the one for the DB index in the sense that they all utilize the absolute meteorological fields but not anomalies in determining an instantaneous measure of the WB direction.

Figure 1a shows a typical case identified through the PV- $\theta$ method (Pelly and Hoskins 2003; Masato et al. 2013) as a blocking event that persisted for 14 days over the northern Far East and western North Pacific after its onset on 8 January 2012. According to the classification method of Masato et al. (2012), this case should be regarded as a cyclonic-cold blocking event, since the DB index (thick blue line in Fig. 1a) is -0.60 at the onset day, while the time-averaged RI index is actually -0.26 . As evident in Fig. 1a, however, the DB index exhibited large day-to-day variations during this event, whereas the RI index underwent a marked increase from its negative values on the first 9 days into its positive values on the following 5 days. In fact, this event was characterized by a blocking low configuration during the first nine days (Fig. 1b) and then by a blocking high configuration afterward (Fig. 1c). The particular event thus demonstrates that both the DB and RI indices can be rather sensitive to when and where WB is occurring. This implies that the method derived by Masato et al. (2012) may obscure the diversity of blocking flow configurations, and thus an improved classification method is needed. It will be shown in section 4 that our new index can indeed separate the aforementioned 14-day period (shown in Fig. 1) into two distinct WB events.

On the basis of the above discussion, the aim of the present study is to propose a new index for identifying WB events with blocking flow configurations. Unlike the many other indices mentioned above that are basically constructed from an instantaneous morphological perspective, our index detects the WB events from a wave-activity perspective. The rest of this paper is organized as follows. After describing our data and new detection algorithms for WB events in section 2, classification and dynamical analysis for WB events are presented in section 3. Section 4 discusses the formation mechanisms for different types of WB events. The final section includes a summary.

\section{Data and methods}

\section{a. Data processing}

In this study, we use daily data from the Japanese 55-year Reanalysis (JRA-55) project conducted by the Japan 
(a)

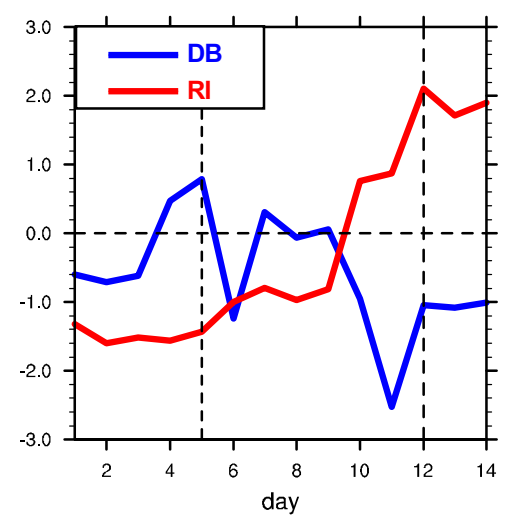

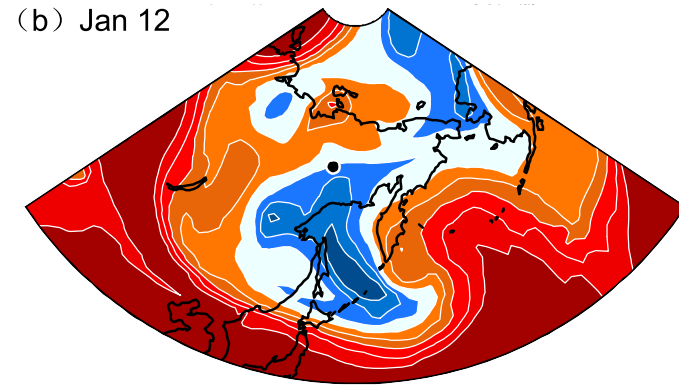

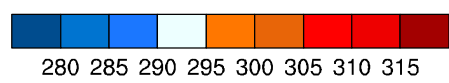

(c) Jan 19
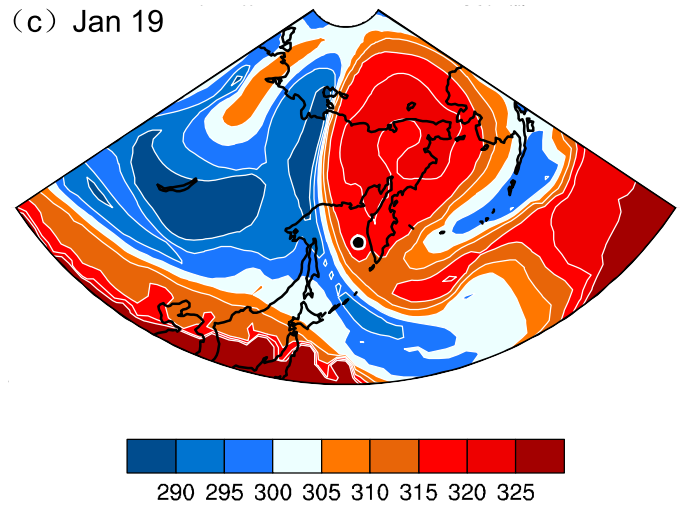

FIG. 1. A typical blocking event that started on 8 Jan 2012 and persisted over 14 days, according to an instantaneous method by Masato et al. (2013). (a) Daily evolution of the DB and RI indices (blue and red solid lines, respectively; as described in the text). Horizontal red dashed line indicates the RI index averaged during the entire event. (b) A map of potential temperature on the 2-PVU surface on 12 Jan 2012. Black dot indicates the wavebreaking center. (c) As in (b), but for 19 Jan 2012. Those days are marked in (a) as vertical black dashed lines.

Meteorological Agency for the period from 1958 to 2014 (Kobayashi et al. 2015). The 6-hourly dataset is available globally on a regular longitude-latitude grid at intervals of $1.25^{\circ}$. Meteorological fields used in the present study include 1) variables on isobaric surfaces, including geopotential height, temperature, and wind velocity, and 2) variables on the $340-\mathrm{K}$ isentropic surface, including PV and wind velocity. Unless otherwise stated, the abovementioned meteorological fields have been low-pass-filtered to remove high-frequency fluctuations associated with migratory transient disturbances so as to extract a slowly varying component with periods longer than 8 days. The influence of the high-frequency disturbances on the low-frequency fluctuations is evaluated, as shown in section $2 \mathrm{~d}$. The daily data are analyzed for the boreal cold season (November-March). A local anomaly of a given variable on a particular date was defined as its instantaneous departure from the climatological-mean annual cycle for the corresponding calendar day. The climatological annual cycle was obtained for a given calendar day as the average of its 31-day (centered) running mean over the $57-y r$ period.

To enhance significance of the composited local anomalies, this study uses the same shifting procedure as employed in Nakamura et al. (1997), which rotate a field along a great circle from a target point to the corresponding reference point. The definitions of both "target point" and "reference point" will be given in section $3 \mathrm{~b}$. To measure the significance of the composite analysis, the false discovery rate (FDR) (Wilks 2016) with the control level $\alpha_{\mathrm{FDR}}=0.05$ is assessed in the two-tailed $t$ test in case of excessive optimism in the multiple hypothesis tests.

\section{b. Detection scheme}

Since the macroscale reversal feature is subtle for detecting WB events (Fig. 1; Altenhoff et al. 2008; Small et al. 2014), the present study focuses only on high-amplitude anomaly events. As argued by Schwierz et al. (2004) and Nishii et al. (2011), it is high-amplitude circulation anomalies in the middle and upper troposphere that characterize salient features of blocking episodes. In addition, high-amplitude anomaly events generally accompany cutoff highs and the macroscale reversal feature of $\theta_{2 \text { PVU }}$ (Nishii et al.2011). Accordingly, the present study identifies each WB event from the following aspects: 1) high amplitude, 2) $\mathrm{WB}$, and 3) blocking flow configuration. In addition, the identification methods for the time evolution and duration of the WB events are described in the sections $2 b(5)$ and $2 b(4)$, respectively.

\section{1) High Amplitude}

The present study follows Nishii et al. (2011) to detect the high-amplitude anomaly feature of WB events by using 
low-pass-filtered geopotential height anomalies at $300 \mathrm{hPa}\left({\mathrm{Z} 300^{\prime}}^{\prime}\right.$ for brevity). According to the positive or negative sign of the primary Z300' (anomaly), the high-amplitude events are classified into two categories: high pressure or low pressure events. Unless otherwise stated, the following takes a high pressure event as an example to explicitly describe the identification algorithm. At first, we identify grid points with a local maximum value of $\mathrm{Z}^{3} 30^{\prime}$ every day in the boreal cold season (November-March) over the 57-yr period. The location of a local maximum of $\mathrm{Z}^{3} 00^{\prime}$ was regarded as the $\mathrm{Z} 300^{\prime}$ center. The peak day of a high-amplitude high pressure event was identified if the local maximum of $\mathbf{Z}^{\prime} 300^{\prime}$ is 1) larger than or equal to its local unit standard deviation and 2) the largest over the region within $1500 \mathrm{~km}$ of the $\mathrm{Z} 300^{\prime}$ center during a 3-day period centered at that peak day. In the following, the $\mathrm{Z} 300^{\prime}$ center on the peak day is referred to as the primary Z300' center. It should be pointed out that the terms "high pressure" and "low pressure" events are analogous to "warm" and "cold" events, respectively, as described by Masato et al. (2012).

\section{2) WAVE BREAKING}

To differentiate our definition from the instantaneous and morphological perspective as in the PV $-\theta$ method, we introduce the concept of WB, which can also be understood dynamically from a wave-activity perspective. For the unforced small-amplitude disturbances superimposed on an unforced basic flow, the density of wave-activity pseudomomentum $A$ and its flux $\mathbf{F}$ approximately satisfy $\partial A / \partial t=-\nabla \cdot \mathbf{F}$. In the Wentzel-Kramers-Brillouin sense, the relationship between the PV flux and divergence of wave-activity flux is obtained as $\nabla \cdot \mathbf{F} \approx p \mathbf{n} \cdot \mathbf{u}^{\prime} q^{\prime}$ without any kind of phase averaging (Takaya and Nakamura 2001), where $p=$ pressure $/ 1000(\mathrm{hPa}), \mathbf{u}^{\prime}=\left(u^{\prime}, v^{\prime}\right)$ denotes small-amplitude wind perturbations superimposed on a steady, zonally inhomogeneous background flow, $\mathbf{n}$ is the local unit vector in the direction of the background PV gradient, and $q^{\prime}$ represents quasigeostrophic PV perturbations. The wave-activity flux $\mathbf{F}$ is closely related to the extended Eliassen-Palm (E-P) flux derived by Trenberth (1986) under the assumption of a zonally uniform basic flow. For the sake of simplicity, $\boldsymbol{v}^{\dagger}=\mathbf{n} \cdot \mathbf{u}^{\prime}$ and $\boldsymbol{v}^{\dagger} q^{\prime}$ will be hereafter referred to as anomalous wind velocity and anomalous PV flux, respectively.

As illustrated later in our examples, a blocking flow configuration tends to be straddled by negative $v^{\dagger} q^{\prime}$ to its upstream flank and positive $v^{\dagger} q^{\prime}$ to its downstream flank. These signreversed PV fluxes can act to induce westward movement of the block by enhancing its western portion and weakening its eastern portion. However, it does not necessarily induce the overall strengthening/weakening of the block unless there is nonzero net PV flux over the entire blocking domain. As was elucidated in the blocking studies by Nakamura (1994), Nakamura et al. (1997), and Nakamura and Fukamachi (2004), the net PV flux is evaluated at the primary anomalous PV center. They pointed out that, at the primary anomalous PV center, negative $v^{\dagger} q^{\prime}$ corresponding to the net convergence of $\mathbf{F}$ means the net accumulation of wave activity into a developing blocking ridge, zero $v^{\dagger} q^{\prime}$ indicates the saturation around its peak day, and then positive $v^{\dagger} q^{\prime}$ corresponds to downstream release of accumulated wave activity out of the decaying blocking ridge.

In the aforementioned studies blocking high events are actually high-amplitude high pressure events according to our definition. The entire processes of WB for a high-amplitude episode can be understood through $v^{\dagger} q^{\prime}$ at the primary anomalous PV center that features the following three dynamical aspects: 1) accumulation, 2) saturation, and 3) release of wave activity, corresponding to the build-up and decay of the highamplitude event in association with the convergence and divergence, respectively, of the wave-activity flux $\mathbf{F}$. The waveactivity perspective thus emphasizes dynamical aspects of WB associated with the anomalous circulation, while the instantaneous methods emphasize the irreversible breaking features of the total (absolute) field.

On the basis of the above argument, our algorithms identify WB as the sign reversal of $v^{\dagger} q^{\prime}$ as follows. For each highamplitude high pressure event, the center of the primary negative PV anomaly on the $340-\mathrm{K}$ isentropic surface is first identified over the region within $1500 \mathrm{~km}$ of the positive $\mathrm{Z}^{3} 00^{\prime}$ center on the peak day. Then, the primary negative PV anomaly center is traced both forward and backward in time under such a constraint that the distance between the primary PV anomaly centers on two adjacent days must be less than $1500 \mathrm{~km}$. The anomalous wind velocity $\boldsymbol{v}^{\dagger}=\mathbf{n} \cdot \mathbf{u}^{\prime}$, which is perpendicular to the local climatological-mean flow, is then determined on the $340-\mathrm{K}$ isentropic surface at the primary $\mathrm{PV}$ anomaly center. Then, WB is considered to occur if the sign of $v^{\dagger} q^{\prime}$ changes temporally from negative to positive at the primary PV anomaly center, which is also applicable to high-amplitude low pressure events. Note that some events are characterized by multiple sign reversals from negative to positive, which is regarded as continual occurrence of WB. Based on the low-pass-filtered field, $v^{\dagger} q^{\prime}$ in this study is associated mainly with quasi-stationary Rossby wave trains. The date of sign reversal of $v^{\dagger} q^{\prime}$, however, may not necessarily coincide with the peak day of a high-amplitude event, especially if occurring under strong feedback forcing from migratory transient eddies (Lau 1988; Nakamura et al. 1997). Thus, the sign reversal date is searched during the 7-day period centered at the peak day of a given highamplitude event.

In fact, wave tilting (longitude vs height, or vs latitude) is essential to the meridional PV flux. In the absence of tilt, a Rossby wave is characterized by meridional wind precisely in quadrature with vorticity and thus the vorticity flux is zero (and more generally the PV flux, too, at the tropopause level). Therefore, nonzero meridional PV flux either involves baroclinic tilt (with height) or barotropic "tilt" on isentropic surfaces, which would occur during a WB event. The sign of the PV flux is determined by the direction of the tilt. This is another reason why $v^{\dagger} q^{\prime}$ is a suitable diagnosis.

\section{3) BLOCKING FLOW CONFIGURATION}

The scheme of Nishii et al. (2011) for detecting blocking flow configurations is adopted here. For a WB high pressure event, as an example, the blocking flow configuration is considered to emerge if a local maximum in the low-pass-filtered total $500-\mathrm{hPa}$ 
height field is detected within $1500 \mathrm{~km}$ of primary $\mathrm{Z} 300^{\prime}$ center in the 3-day period centered at the peak time of the WB event. In the following, a high-amplitude high pressure WB event with a blocking flow configuration will be referred to as a WBBH event for the sake of brevity. Analogously, a low pressure WB event will be referred to as a WBBL event.

\section{4) EvOlving FEATURES}

As suggested by Tyrlis and Hoskins (2008b) and Masato et al. (2012), different types of blocking exhibit distinct features in the zonal movement of the primary PV center, which depend on the shear of the background westerlies. Taking a blocking high as an example, its formation can be initiated with a poleward intrusion of low-PV air from lower latitudes. Under the anticyclonic background westerly shear, the low-PV air tends to move northeastward and the blocking ridge tends to tilt in the southwest-northeast direction, which has been referred to as the anticyclonic wave breaking. In contrast, under the cyclonic background westerly shear, the low-PV air tends to move northwestward and the blocking ridge thus tends to tilt in the southeast-northwest direction, which is usually referred to as the cyclonic wave breaking. Further discussion on the linkage between our eastward/westward types and the anticyclonic/cyclonic types is given in section 4 .

Here, a particular feature of an evolving WBBH event is highlighted with the longitudinal movement of the primary PV anomaly center from day -3 to day $3, \Delta \lambda=\lambda_{\text {day } 3}-\lambda_{\text {day }-3}$. Hereafter, for brevity, day $-N(+N)$ refers to the day that is $N$ days before (after) the peak day of a WBBH event. As shown in section $3 \mathrm{a}$, the characteristic time scale of $\mathrm{WB}$ events is approximate 9 days. If $\Delta \lambda \geq 10^{\circ}$, then the $\mathrm{WBBH}$ is regarded as the eastward type. If $\Delta \lambda \leq-10^{\circ}$, in contrast, it is regarded as the westward type. The rest is referred to as the stationary type that tends to show more stationary feature in the PV field around the peak day than the two other types. For a WBBH event whose start and end days cannot be identified within three days before and after its peak day, $\Delta \lambda$ is obtained as $\lambda_{\text {end }}$ day $-\lambda_{\text {start_day }}$ and $\Delta \lambda$ /duration $=1.5^{\circ}$ day $^{-1}$ is set as the criterion for its classification. Sensitivity of the classification to the choice of $\Delta \lambda$ has been tested by taking other threshold values around $10^{\circ}$ or other period lengths such as 3 days (from day -1 to day 1 ) and 11 days (from day -5 to day 5 ). We have confirmed that the final results are qualitatively unchanged.

A WBBL event can be identified in an analogous manner by searching a local minimum of the low-pass-filtered total 500$\mathrm{hPa}$ height field within $1500 \mathrm{~km}$ around the primary negative $\mathrm{Z}^{300^{\prime}}$ center. The WBBL is also further classified into the eastward or westward types if $\Delta \lambda \geq 10^{\circ}$ and $\Delta \lambda \leq-10^{\circ}$, respectively, or otherwise stationary type.

\section{5) Duration}

The duration for a given WBBH event is defined as the period from its start day to its end day. The start and end days are defined as the first and last days, respectively, when the value of $\mathrm{Z} 300^{\prime}$ at the center exceeds its counterpart at the primary $\mathrm{Z}_{300}$ center at the peak day divided by $e(\approx 2.718)$. To focus on quasi-stationary anomaly events, we also require that for a given event, its $\mathrm{Z} 300^{\prime}$ centers between two adjacent days must be less than $1500 \mathrm{~km}$ apart, and the $\mathrm{Z} 300^{\prime}$ centers at any given day are within $2500 \mathrm{~km}$ of the primary $\mathrm{Z} 300^{\prime}$ center at the peak day. The duration for a given WBBL event is determined in an analogous manner.

\section{c. Wave-activity flux}

In the present study, $v^{\dagger} q^{\prime}$ is evaluated at the location of the PV anomaly center to represent the net local divergence/ convergence of the wave-activity flux around the PV anomaly center. Here, we use the wave-activity flux itself to further verify whether the WB events are accompanied by welldefined propagation of Rossby wave trains and whether the flux convergence/divergence is consistent with the sign of $v^{\dagger} q^{\prime}$. The wave-activity flux for quasigeostrophic eddies derived by Takaya and Nakamura (2001) is employed in the following. The flux on an isobaric surface may be expressed as follows:

$$
\mathbf{F}=\frac{p}{2|\mathbf{U}|}\left(\begin{array}{l}
U\left(\psi_{x}^{2}-\psi^{\prime} \psi_{x x}^{\prime}\right)+V\left(\psi_{x}^{\prime} \psi_{y}^{\prime}-\psi^{\prime} \psi_{x y}^{\prime}\right) \\
U\left(\psi_{x}^{\prime} \psi_{y}^{\prime}-\psi^{\prime} \psi_{x y}^{\prime}\right)+V\left(\psi_{y}^{\prime 2}-\psi^{\prime} \psi_{y y}^{\prime}\right) \\
\frac{f_{0}^{2}}{N^{2}}\left[U\left(\psi_{x}^{\prime} \psi_{z}^{\prime}-\psi^{\prime} \psi_{x z}^{\prime}\right)+V\left(\psi_{y}^{\prime} \psi_{z}^{\prime}-\psi^{\prime} \psi_{y z}^{\prime}\right)\right]
\end{array}\right)+\mathbf{C}_{\mathbf{U}} M
$$

where $p=$ pressure $/ 1000(\mathrm{hPa}), \mathbf{U}=(U, V, 0)$ is the basic flow, $f_{0}$ is the Coriolis parameter at $45^{\circ} \mathrm{N}, \psi$ is the streamfunction, $N^{2}$ is the buoyancy frequency squared, $\mathbf{C}_{\mathbf{U}}$ is the wave phase velocity in the direction of $\mathbf{U}$, and $M$ is wave-activity density. The primes represent low-frequency perturbations and the subscripts partial derivatives. As demonstrated in Nishii et al. (2011) and section 3a, high-amplitude WB events are generally associated with quasi-stationary anomalies (i.e., $\mathbf{C}_{\mathbf{U}} \approx 0$ ). Thus, we neglect the term $\mathbf{C}_{\mathbf{U}} M$ in (1). Theoretically, the flux is independent of wave phase and parallel to the local group velocity of a stationary Rossby wave train in the Wentzel-KramersBrillouin sense. Therefore, the flux is useful for illustrating qualitative aspects of the propagation of a Rossby wave train in a zonally varying climatological-mean flow.

In this study, low-frequency perturbations signified with primes in (1) are taken from the composited daily low-passfiltered anomalies associated with WB events, and $\mathbf{U}$ from the climatological-mean seasonal cycle as described in section 2 a. The composited circulation anomalies can extract typical circulation anomalies coherent among the WB events around a given location, while eliminating anomalies incoherent among the events. Then, the wave-activity flux derived from the composited anomalies can exhibit a typical wave packet propagation during WB events occurring around a given location.

As discussed by Wolf and Wirth (2017), due to the semigeostrophic nature of Rossby waves, the wave-activity flux is, strictly speaking, not phase independent. In the following analysis we therefore focus on the net flux convergence/ divergence around the primary PV anomaly center rather than its fine details. Although the wave-activity flux $\mathbf{F}$ is better than other conventional methods in detecting the Rossby wave trains, care must be exercised in interpreting the flux, since it is 
valid only for weakly nonlinear waves. To analyze strongly nonlinear waves, one can use other diagnostics (Huang and Nakamura 2016; Wolf and Wirth 2017).

\section{d. Transient eddy feedback forcing}

As shown in Nakamura et al. (1997), both local breaking of quasi-stationary Rossby wave trains and transient eddy feedback forcing (TEFF) can contribute to the formation of highamplitude high pressure events (often referred to as blocking highs), and their relative importance appears to be of regional dependence. Those events over continental regions (e.g., Europe) tend to be characterized by local breaking of quasistationary Rossby wave trains, while those events over maritime regions (e.g., North Pacific) tend to develop under strong TEFF and thus the associated $v^{\dagger} q^{\prime}$ around the amplifying anomaly center is nearly zero.

For the present study, both barotropic and baroclinic components of the feedback forcing from high-frequency migratory eddies have been evaluated through the following geopotential height tendency equation (Lau and Holopainen 1984; Lau and Nath 1991):

$$
g\left[\frac{1}{f} \nabla^{2}+f \frac{\partial}{\partial p}\left(\frac{1}{\sigma} \frac{\partial}{\partial p}\right)\right] \frac{\partial Z}{\partial t}=\underbrace{-\nabla \cdot \overline{\mathbf{u}^{*} \boldsymbol{s}^{*}}}_{D^{\text {vort }}}+\underbrace{f \frac{\partial}{\partial p}\left(\frac{\nabla \cdot \overline{\mathbf{u}^{*} \theta^{*}}}{\tilde{S}}\right)}_{D^{\text {heat }}},
$$

where asterisks and overbars denote high-pass- and low-passfiltered quantities, respectively, with a cutoff period of 8 days; $\mathbf{u}=(u, v), \tilde{S}$ denotes the hemispheric mean of $-\partial \bar{\theta} / \partial p ; \sigma=$ $-(1 / \rho \theta)(\partial \theta / \partial p)$ as a static stability parameter, which is assumed to be a function of pressure $p$ only; and other notations are standard. In (2), $D^{\text {vort }}$ represents the convergence of eddy vorticity flux, and $D^{\text {heat }}$ the eddy heat flux convergence. As the solutions of (2) separately with $D^{\text {vort }}$ and $D^{\text {heat }}$, one can obtain the barotropic and baroclinic components, respectively, of local TEFF in the form of low-frequency tendencies of geopotential height $(\partial Z / \partial t)$ (Lau and Holopainen 1984; Lau and Nath 1991; Nishii et al. 2009).

In solving (2), the boundary conditions were set at the 100and $1000-\mathrm{hPa}$ levels as follows:

$$
\left\{\begin{array}{l}
-\frac{g p}{R}\left(\frac{p_{0}}{p}\right)^{R / C_{p}} \frac{\partial}{\partial p}\left(\frac{\partial Z}{\partial t}\right)_{\text {heat }}=-\nabla \cdot \overline{\mathbf{v}^{*} \theta^{*}} \\
\frac{\partial}{\partial p}\left(\frac{\partial Z}{\partial t}\right)_{\text {vor }}=0
\end{array}\right.
$$

The spherical harmonics with the T21 truncation are used for solving (2), which has been documented in detail by Holopainen and Fortelius (1987) and by Takaya and Nakamura (2005).

\section{Results}

\section{a. Frequency distribution and duration}

Figure 2 shows spatial distributions of the primary Z300' centers at the peak day for both the WBBH and WBBL events observed in the 57 cold seasons. A local value represents the total number of those WB events identified within $500 \mathrm{~km}$ of the particular grid point. At any grid point, if the peak-day interval between the two adjacent events is less than 8 days, the event with smaller amplitude has been discarded. As evident in Figs. $2 \mathrm{a}$ and $2 \mathrm{~b}$, both $\mathrm{WBBH}$ and $\mathrm{WBBL}$ events tend to occur frequently over the North Atlantic, the Eurasian continent, Northeast Asia, the central and eastern North Pacific, and North America. Compared to the WBBH events, WBBL events tend to be more frequent except over the Arctic Ocean, and the frequency is particularly high over the North Atlantic, Europe, western Russia, central Siberia/Asia, East Asia, the central/eastern North Pacific, and Hudson Bay. In these active regions, the frequency exceeds 25 , even reaching 45 at some locations, which means that extreme wintertime WBBL events are observed on average once every other winter over many areas in the extratropical Northern Hemisphere.

In Fig. 2, thick black and blue lines mark locations at which $50 \%$ and $90 \%$, respectively, of the total high-amplitude WB events exhibit blocking flow configurations. The percentage generally increases with latitude. Among the WBBH events in particular (Fig. 2a), high-amplitude WB events in the polar region are more likely to exhibit blocking flow configurations than in their midlatitude counterparts. Therefore, our results also confirm that WB events defined from the wave-activity perspective are not necessarily classified into blocking events.

Previous studies have revealed that the climatological frequency of blocking highs in boreal winter tends to be particularly high over three regions: the North Atlantic, the North Pacific, and the Ural Mountains (e.g., Pelly and Hoskins 2003; Diao et al. 2006; Croci-Maspoli et al. 2007; Tyrlis and Hoskins 2008a; Barriopedro et al. 2010). However, different methods can lead to distinct frequency distributions of blocking highs over the North Atlantic and North Pacific (Woollings et al. 2008; Barriopedro et al. 2010; Dunn-Sigouin et al. 2013; Pinheiro et al. 2019). The "absolute-field" method tends to detect more blocking days over the Euro-Atlantic sector than over the Pacific, and higher frequency over the eastern Atlantic/ Europe than over the central Atlantic, as well as over the western/ central Pacific than over the eastern Pacific (e.g., Tibaldi and Molteni 1990; Pelly and Hoskins 2003; Tyrlis and Hoskins 2008a). In contrast, the "departure-field" method tends to show the opposite tendency (e.g., Dole and Gordon 1983; Shukla and Mo 1983).

Figure $2 \mathrm{a}$ indicates a tendency for WBBH events to occur preferentially over the North Atlantic/Europe, the North Pacific, and the Ural Mountains, with higher frequency in the North Atlantic than in the North Pacific. The central Atlantic and eastern Pacific are the most active areas within the respective ocean basins. Thus, our results seem to be a mixture of the frequency distributions of blocking highs obtained by those two methods, which is consistent with other combined absolute-departure methods (e.g., Croci-Maspoli et al. 2007; Barriopedro et al. 2010; Dunn-Sigouin et al. 2013), except for relatively few occurrences over the eastern North Pacific. Compared to their counterpart over the Atlantic, fewer highamplitude high pressure events over the eastern Pacific exhibit both WB signature and blocking flow configuration through our detection (not shown). By contrast, the local maximum around the Ural Mountains (Fig. 2a) seems to be insensitive to 


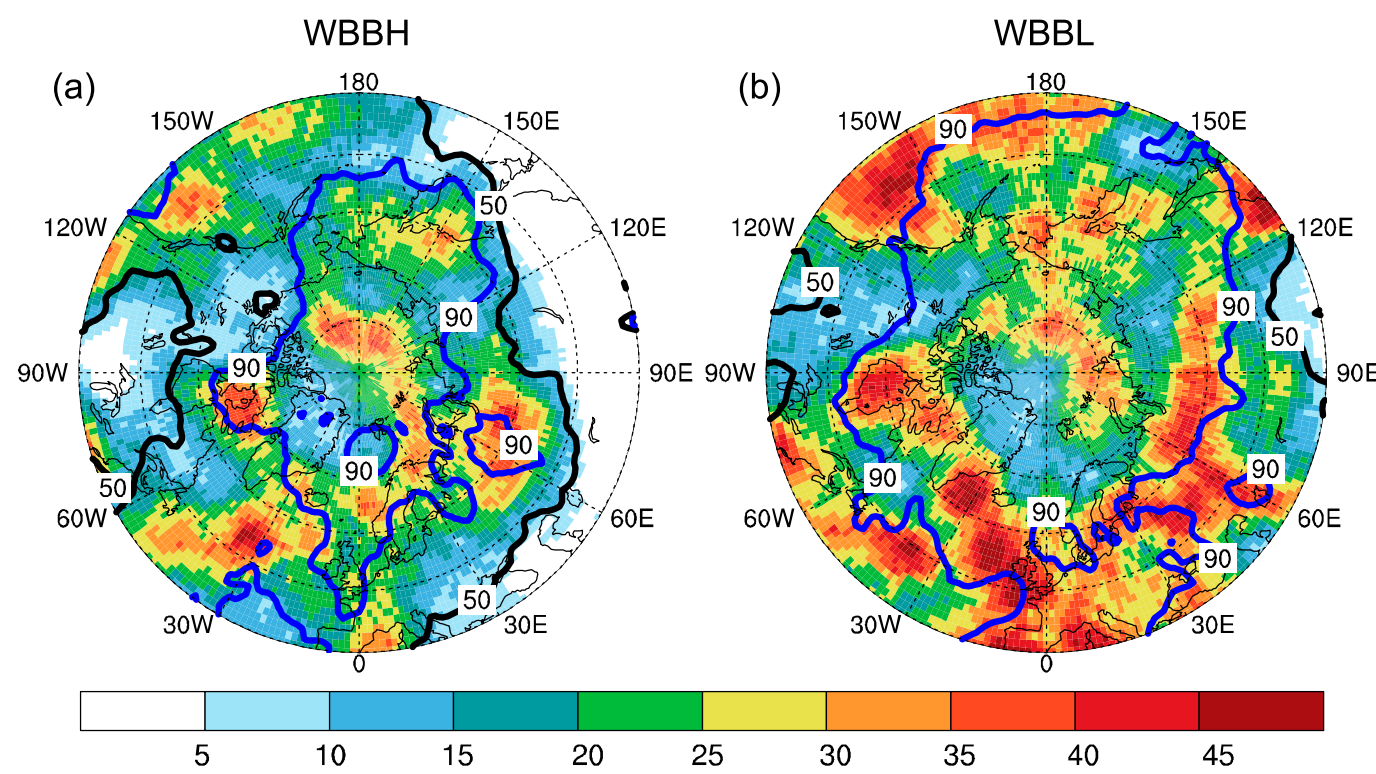

FIG. 2. (a) Spatial distribution of frequency of primary Z300' centers for WBBH events (with blocking flow configurations) at the peak day. Value at a given location represents the total number of events identified within $500 \mathrm{~km}$ in the 57 cold seasons. Locations at which $90 \%(50 \%)$ of the total high-amplitude WB events exhibit blocking flow configurations, regarded as WBBH events, are marked with thick blue (black) contours. (b) As in (a), but for WBBL events.

detection methods. In fact, both the absolute-field method (e.g., Tibaldi and Molteni 1990) and the departure-field method (e.g., Dole and Gordon 1983) can identify the local maximum or the relative high frequency around the Ural Mountains, which has also been verified by recent studies (e.g., Woollings et al. 2008; Barriopedro et al. 2010; Pinheiro et al. 2019) through comparison of the different detection methods. Our results are overall consistent with the previous studies, although the present study emphasizes both WB signatures and blocking flow configurations. In addition, WBBH events also tend to occur rather frequently over the Arctic Ocean, which was not documented in most of the previous studies in which so-called reference latitudes are prescribed for blocking detection. Our algorithm, in contrast, requires no reference latitude and is therefore capable of detecting blocking highs even in the polar region.

The distribution of blocking lows in the wintertime Northern Hemisphere obtained by Dole and Gordon (1983) is quite similar to its counterpart for the blocking highs, featuring the North Pacific, the North Atlantic, and the Ural Mountains as the three preferred regions for their occurrence. This resemblance in frequency distribution between the blocking highs and lows is analogously observed in our statistics between the WBBHs (Fig. 2a) and WBBLs (Fig. 2b), except for the overall higher frequency of the WBBLs.

Persistence of $\mathrm{WBBH}$ and $\mathrm{WBBL}$ events is compared in Fig. 3. As seen in Figs. $3 a$ and 3c, common events that exceed $5 \%$ in frequency are found to have durations between 7 and 14 days. The most common WBBH (WBBL) events, which exceed $8 \%(6 \%)$ in frequency, have the duration of 9 days (Fig. 3a). In addition, the characteristic time scale of the events can also be explored through the semilog plots (Figs. 3b,d). As demonstrated by Dole and Gordon (1983), Pelly and Hoskins (2003), and Barriopedro et al. (2010), blocking events are characterized by a first-order Markov process, where the probability of an event that lasts $n$ days and survives to $n+$ 1 days is independent of $n$. The logarithmic profile of blocking histogram can be fitted with a straight line whose slope represents a characteristic time scale of blockings. Figures $3 \mathrm{~b}$ and $3 \mathrm{~d}$ show semilog plots of the cumulative histogram (i.e., the number of WB episodes with durations equal or greater than a given bin). Obviously, the deceasing exponential curve seems almost linear for the WB events, if we focus only on the episodes with midrange durations from 9 to 25 days and exclude the noisy tail ends of the distribution. The reciprocal of the slope of the linear regression is approximate 8.8 days for WBBH events and 8.7 days for WBBL events. Therefore, 9 days can be regarded as a characteristic time scale of the highamplitude WB events.

Figure 4 shows the classification of the high-amplitude WB events (Fig. 2) according to the zonal displacement of the primary PV anomaly center. On the whole, WBBHs are dominated by the eastward type that mainly occur over the northern North Atlantic, northern Europe, western/central Siberia, and the eastern Arctic Ocean (Fig. 4a). By contrast, WBBH events of the westward type are less frequent and characterized by retrogression of a blocking high that occurs mainly over a zonally elongated region around the Labrador Sea, Iceland, the central Arctic Ocean, and eastern Siberia (Fig. 4b). In fact, Takaya and Nakamura (2005) and Nishii et al. (2010) found that a blocking high that develops north of the Okhotsk Sea tends to evolve westward under strong TEFF. 

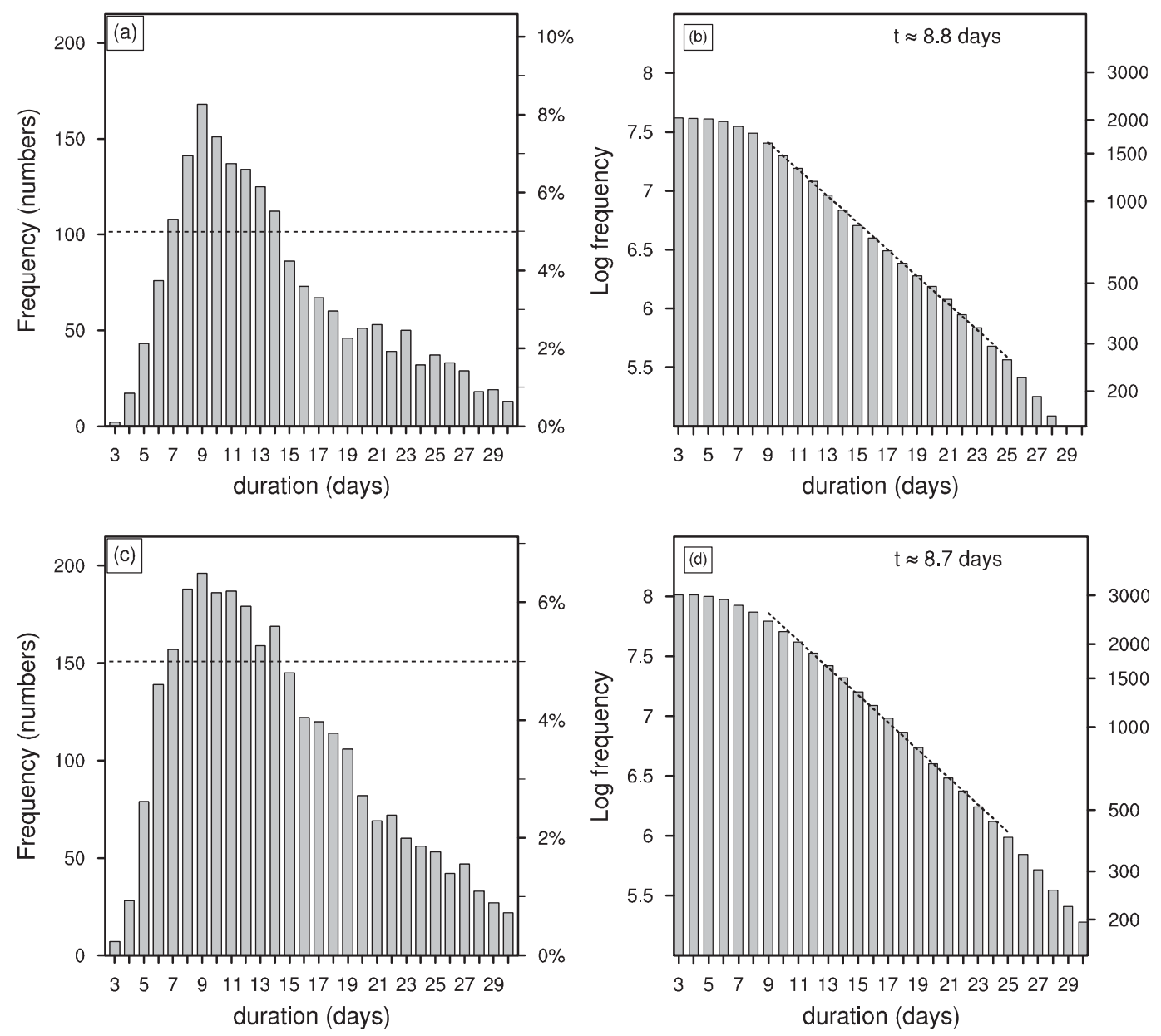

FIG. 3. (a) Number of WBBH events lasting a given number of days. (b) As in (a), but the log (to base $e$ ) of cumulative numbers. (c),(d) As in (a) and (b), respectively, but for WBBL events. The right ordinate in (a) and (c) indicates the fraction of the events lasting a given number of days to the total events, and the dashed lines indicate $5 \%$. The right ordinate in (b) and (d) indicates the cumulative numbers. The dashed lines in (b) and (d) indicate linear regressions for blocking episodes with durations from 9 to 25 days.

Tyrlis and Hoskins (2008b) also found that blocking highs tend to retrogress over eastern Siberia and around Iceland. WBBH events of the stationary type are even less frequent (Fig. 4c).

Meanwhile, WBBL events are also mostly of the eastward type, and they tend to occur over the northern North Atlantic, Siberia, midlatitude East Asia, the North Pacific, and Hudson Bay (Fig. 4d). WBBL events of the westward type occur less frequently with much lower maximum values in frequency over the Arctic Ocean and Europe (Fig. 4e). WBBL events of the stationary type are also less frequent, and they tend to occur farther south over the Mediterranean region and the central/eastern North Pacific (Fig. 4f) compared to the eastward type. Due to the relative low frequency over the 57 cold seasons, composited evolution of the stationary-type categories and westward-type WBBL events will not be presented in the following.

\section{b. Composite analysis}

Different types of WB events tend to exhibit different evolving features (e.g., Thorncroft et al. 1993; Peters and Waugh
1996; Tyrlis and Hoskins 2008b; Masato et al. 2013). Moreover, from the dynamical perspective, the influences of both local breaking of quasi-stationary Rossby wave trains and TEFF on the blocking formation also exhibit regional dependence (Nakamura et al. 1997). Thus, it is necessary to compare both the evolution and dynamical features between the different types of WB events. In this subsection, composite analysis is applied for different types of WB events to determine whether they tend to accompany incipient Rossby waves or not. Moreover, the contribution from TEFF is also evaluated to obtain a comprehensive picture on the dynamical features for WB events.

Before the compositing, the shifting procedure (Nakamura et al. 1997) has been applied to circulation anomaly fields along the great circle from a "target point" to a "reference point" for each of the WB events identified around a given location. The reference point is taken as the location with the maximum number of those events, which is marked by the black dots in a given panel of Fig. 4. The target point is the grid point at which 


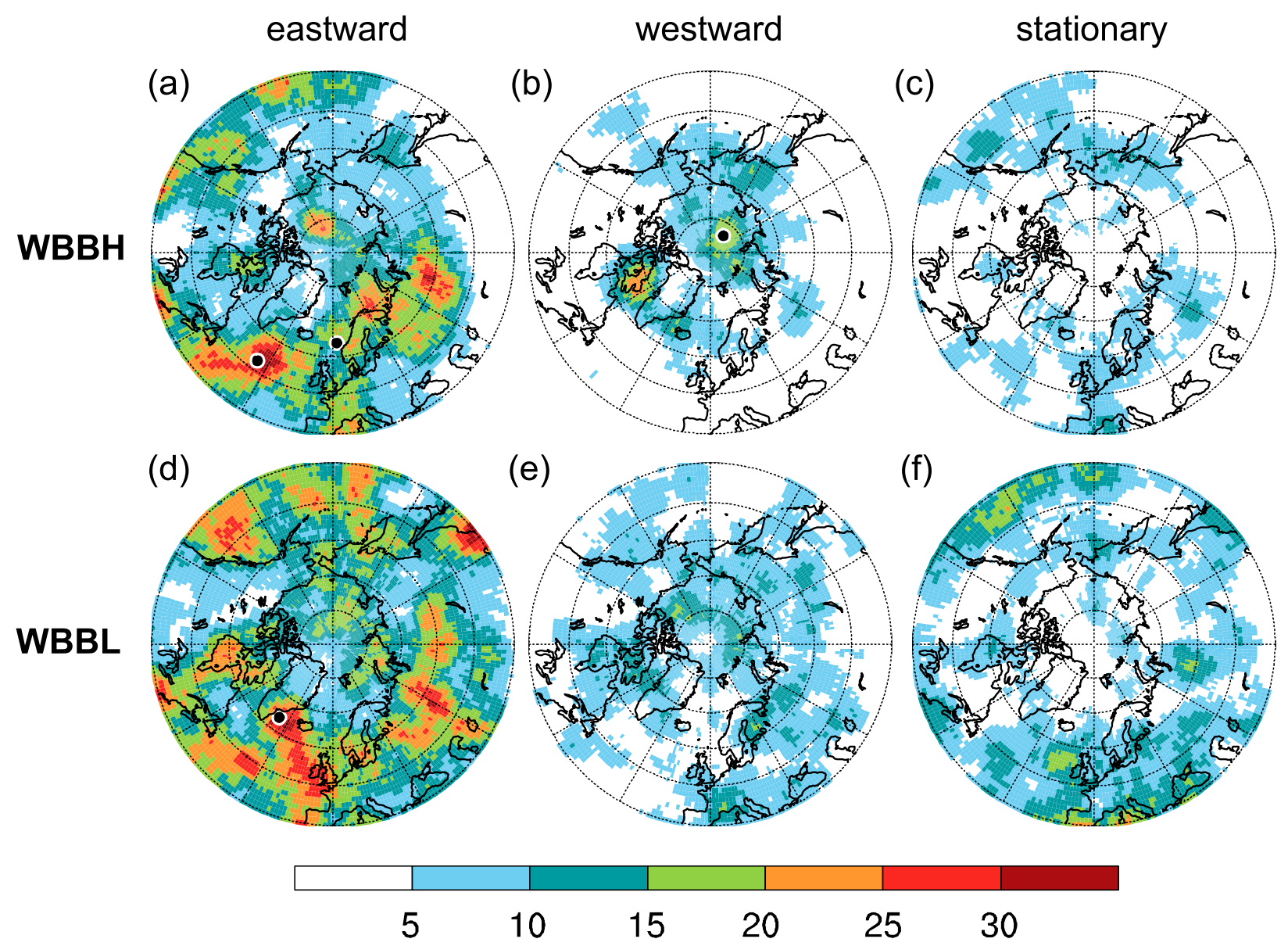

FIG. 4. Frequency distributions of the center positions of various types of high-amplitude WB events with a blocking configuration over the 57 winters: (a)-(c) WBH events and (d)-(f) WBL events. The columns represent the (left) eastward, (center) westward, and (right) stationary types, respectively. The filled black dots in (a), (b), and (e) indicate the grid points where the composite analysis is applied in the following text.

their primary $\mathrm{Z}_{300}$ centers at their peak day are located within $500 \mathrm{~km}$ of the corresponding reference point. It should be stressed that for each of the composites the same spatial shifting is applied to every lag, in order to avoid introducing any artificial distortion in the composited evolution. Therefore, this shifting should never affect our classification of a given WB event as an eastward, westward, or stationary type.

\section{1) EASTWARD-TYPE WBBH EVENTS}

Figure 5 shows the composited evolution of 37 eastwardtype WBBH events in the northern North Atlantic. In the developing stage at day -2 , the primary negative PV anomaly stays under the enhanced southerlies $\left(v^{\dagger}>0\right.$; Fig. $\left.5 \mathrm{~g}\right)$ on the upstream flank of the amplifying pressure ridge (Fig. 5b). The anomalous PV flux is thus overall negative $\left(v^{\dagger} q^{\prime}<0\right)$, which is dynamically consistent with the converging wave-activity flux from the upstream (blue shading in Fig. 5b) into the vicinity of the amplifying PV anomaly center (Fig. 5g). In the mature stage at day 0 (Fig. 5h), the anomalous PV flux is diminished as the PV anomaly center is now located where $v^{\dagger}=0$.
Correspondingly, virtually no net flux divergence appears around the PV anomaly center, as characterized by the converging and diverging wave-activity flux from its upstream and toward downstream, respectively (Fig. 5c). In the decay stage at days +2 and +4 , the anomalous PV flux turns into positive $\left(v^{\dagger} q^{\prime}>0\right)$ as the primary PV anomaly center moves into the region of anomalous northerlies $\left(v^{\dagger}<0\right.$; hatched in Figs. $5 \mathrm{i}$ and $5 \mathrm{j})$. This is dynamically consistent with the diverging waveactivity flux out of the vicinity of the decaying PV anomaly (Figs. 5d,e). In the total height field, the overturning feature as the characteristics of WB and a blocking flow configuration in geopotential height contours are most apparent at day +2 (Fig. 5d).

We emphasize that TEFF also plays an important role in the developing stage. From day -4 to day -2 (Figs. $5 \mathrm{k}, 1$ ), a positive $\mathrm{Z} 300^{\prime}$ tendency with the amplitude as much as $+80 \mathrm{~m} \mathrm{day}^{-1}$ is observed around the negative PV anomaly center. Over the same region, TEFF has induced the significant anomalous height tendency as much as $+24 \mathrm{~m} \mathrm{day}^{-1}$ (Figs. 5p-r). Thus, TEFF can account for $30 \%$ of the observed amplification of the 


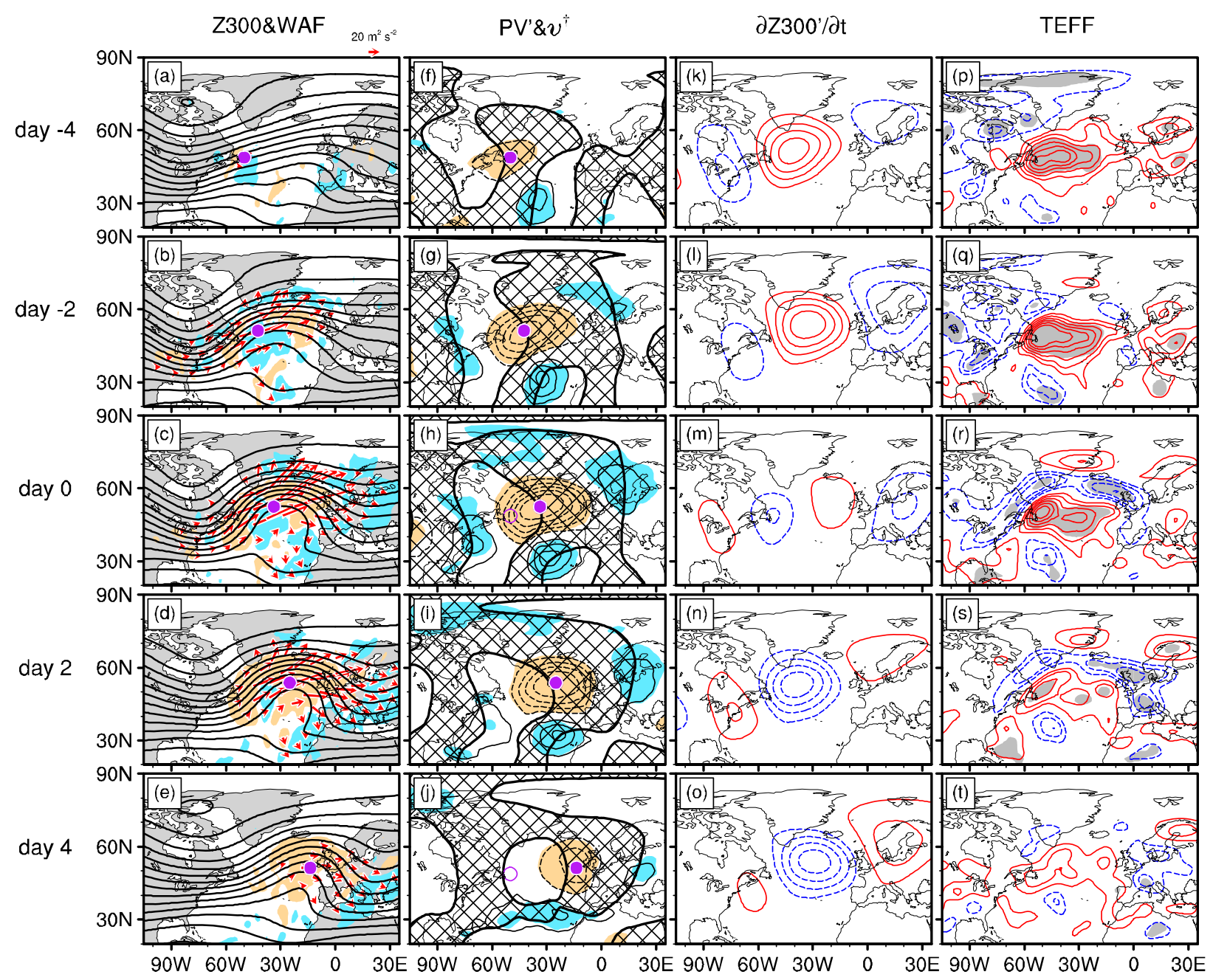

FIG. 5. Composite evolutions of (a)-(e) geopotential height and wave-activity flux (WAF) at $300 \mathrm{hPa}$, (f)-(j) PV anomaly and anomalous wind velocity $\left(v^{\dagger}\right)$ perpendicular to the local climatological mean on the 340-K surface, (k)-(o) observed anomalous 300-hPa height tendency as half the composite difference between the adjacent lags, and (p)-(t) anomalous 300-hPa height tendency induced as TEFF, based on 37 eastward-type WBBH events at $52.5^{\circ} \mathrm{N}, 325^{\circ} \mathrm{E}$ over the 57 winter seasons. In (a)-(e), purple dots indicate the primary low-PV anomaly center at a given lag, while purple open circles in (h) and (j) denote the corresponding center at day -4 . Blue and orange shading represents the convergence and divergence of WAF in (a)-(e), respectively. In (f)-(j), blue and orange shading represents positive and negative PV anomalies, respectively, that are significant at the 5\% level. Gray shading in (p)-(t) represents significant TEFF anomalies at the $5 \%$ level. Hatching in (f)-(j) indicates the equatorward anomalous wind $\left(v^{\dagger}<0\right)$. Contour intervals are $100 \mathrm{~m}$ in (a)-(e), 4 $\times 10^{-7} \mathrm{~K} \mathrm{~m}^{2} \mathrm{~kg}^{-1} \mathrm{~s}^{-1}$ in (f)-(j), $20 \mathrm{~m} \mathrm{day}{ }^{-1}$ in (k)-(o), and $4 \mathrm{~m} \mathrm{day}^{-1}$ in (p)-(t). WAF with amplitude less than $10 \mathrm{~m}^{2} \mathrm{~s}^{-2}$ in (a)-(e), contours of zero PV anomalies in(f)-(j), and zero contours in (k)-(t) are omitted. In each panel, the southernmost latitude is $20^{\circ} \mathrm{N}$.

primary anticyclonic (negative PV) anomaly, as an important contributor to the initiation of the blocking development. After day 0 , TEFF weakens but still remains positive (Figs. $5 \mathrm{~s}, \mathrm{t}$ ), indicating that the emanation of a stationary Rossby wave train is mainly responsible for the decaying of the WBBH events.

One may notice that $v^{\dagger} q^{\prime}$ is positive at day -4 but the waveactivity flux converges around the primary PV center (Figs. 5a,f). In fact, signature of incoming Rossby wave train has not emerged before day -4 (Fig. 5a). At day -3 (not shown), the wave train signature becomes evident, and then $v^{\dagger} q^{\prime}$ becomes negative at the primary PV anomaly center, corresponding to the enhanced net convergence of wave-activity flux. Therefore, the inconsistency between the sign of $v^{\dagger} q^{\prime}$ and the divergence/ convergence of wave-activity flux at day -4 may be associated with the weak signature of incoming Rossby wave trains under the strong TEFF.

Nakamura et al. (1997) revealed that the signature of incoming Rossby wave propagation is clear during the developing stage of a blocking high (actually a high-amplitude event according to our definition) over northern Europe. To confirm their result, the composite analysis is also performed for 23 eastward-type WBBH events observed over northern Europe $\left(62.5^{\circ} \mathrm{N}, 13.75^{\circ} \mathrm{E}\right.$; Fig. 6). Analogous to the eastward-type WBBH events over the northern North Atlantic (Fig. 5), the 


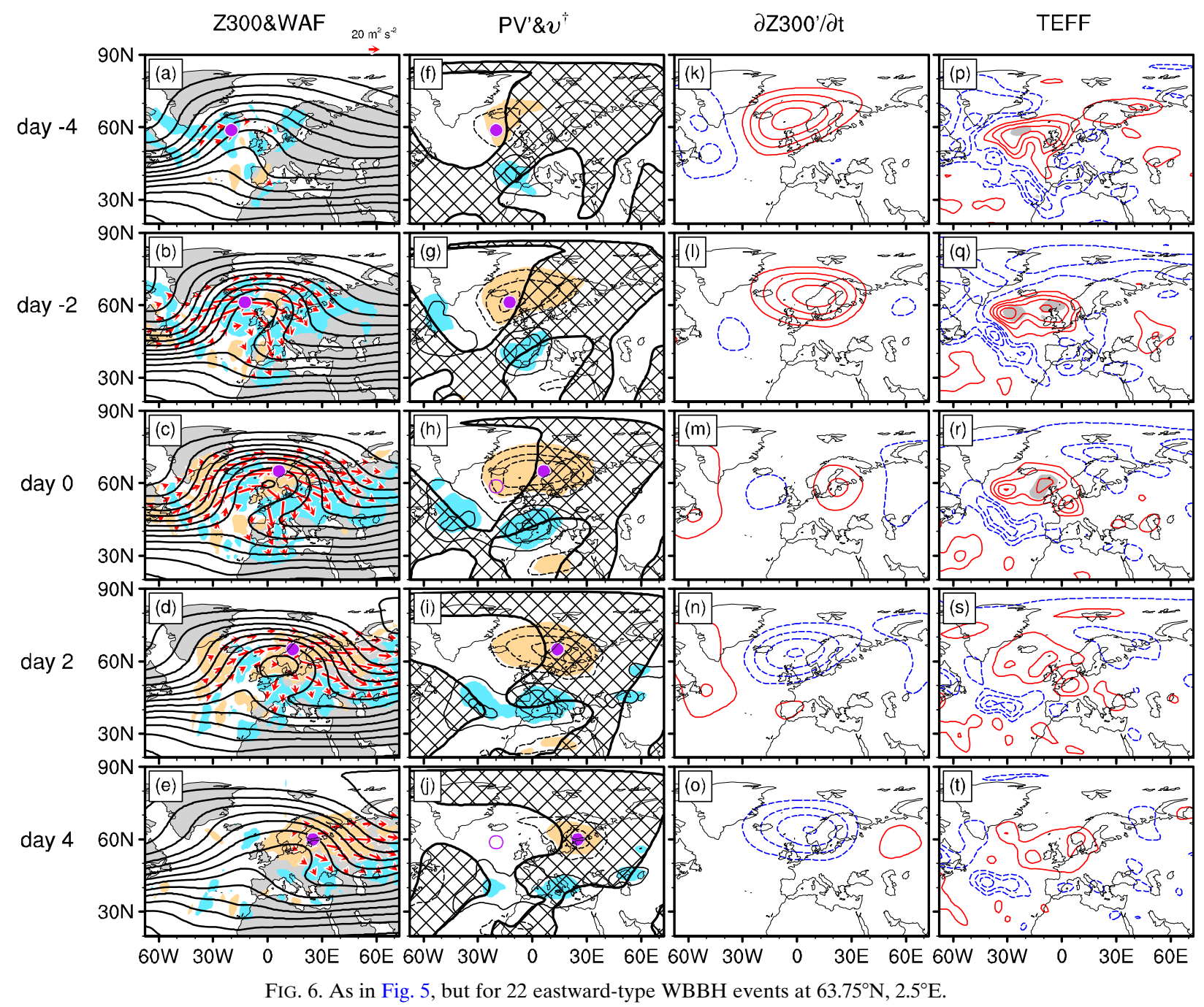

anomalous PV flux is also consistent with the propagation of a Rossby wave train from the North Atlantic. Specifically, corresponding to the sign reversal of $v^{\dagger} q^{\prime}$ around the primary negative PV anomaly center from negative before day 0 (Figs. 6f,g) to positive after day 0 (Figs. 6h-j), the wave-activity flux around the primary PV anomaly center gradually turns from convergent (Figs. 6a,b) to divergent (Figs. 6c-e). Compared to the northern North Atlantic events (Fig. 5), the eastward-type WBBH events over northern Europe thus tend to exhibit an even clearer signature of an incoming Rossby wave train before day 0 (Figs. 6a,b) that emanates from the western North Atlantic at day -4 (Fig. 6a). Moreover, during the life cycle of the WBBH events over northern Europe away from the Atlantic storm track, TEFF is rather modest and only marginally significant (fourth column of Fig. 6), suggestive of large case-to-case variability. Over northern Europe, the composited evolution of the WBBH events thus bears striking similarities to the strong blocking highs depicted by Nakamura et al. (1997), in which local breaking of stationary Rossby wave train plays an essential role.

\section{2) WESTWARD-TYPE WBBH EVENTS}

Figure 7 shows the composited evolution of 23 westwardtype WBBH events over the Arctic Ocean off eastern Siberia. At day -4 (Fig. 7a), a pressure ridge is developing around the Bering Strait to the northeast of a climatological planetarywave trough over eastern Siberia. The ridge exhibits a marked overturning feature already in the developing stage (Fig. 7b). Correspondingly, the negative PV anomaly center is located in the weak anomalous southerlies $\left(v^{\dagger}>0\right)$ on the southwestern flank of the ridge (Figs. 7f,g), yielding negative anomalous PV flux $\left(v^{\dagger} q^{\prime}<0\right)$ locally (Figs. 7a,b,f,g) in a manner consistent with the local convergence of the wave-activity flux (Fig. 7a). Takaya and Nakamura (2005) found that blocking highs develop over the Bering Sea under locally strong TEFF and the origin of the wave-activity flux converging into the ridge may also reflect TEFF. In fact, TEFF acts to reinforce the ridge by inducing a significant positive $\mathrm{Z} 300$ ' tendency as much as $+12 \mathrm{~m} \mathrm{day}^{-1}$ over the Arctic Ocean (Figs. $7 \mathrm{p}-\mathrm{r}$ ), which accounts for $\sim 20 \%$ of the observed amplification of the primary positive height anomalies in the developing stage (Figs. 7k,1). 


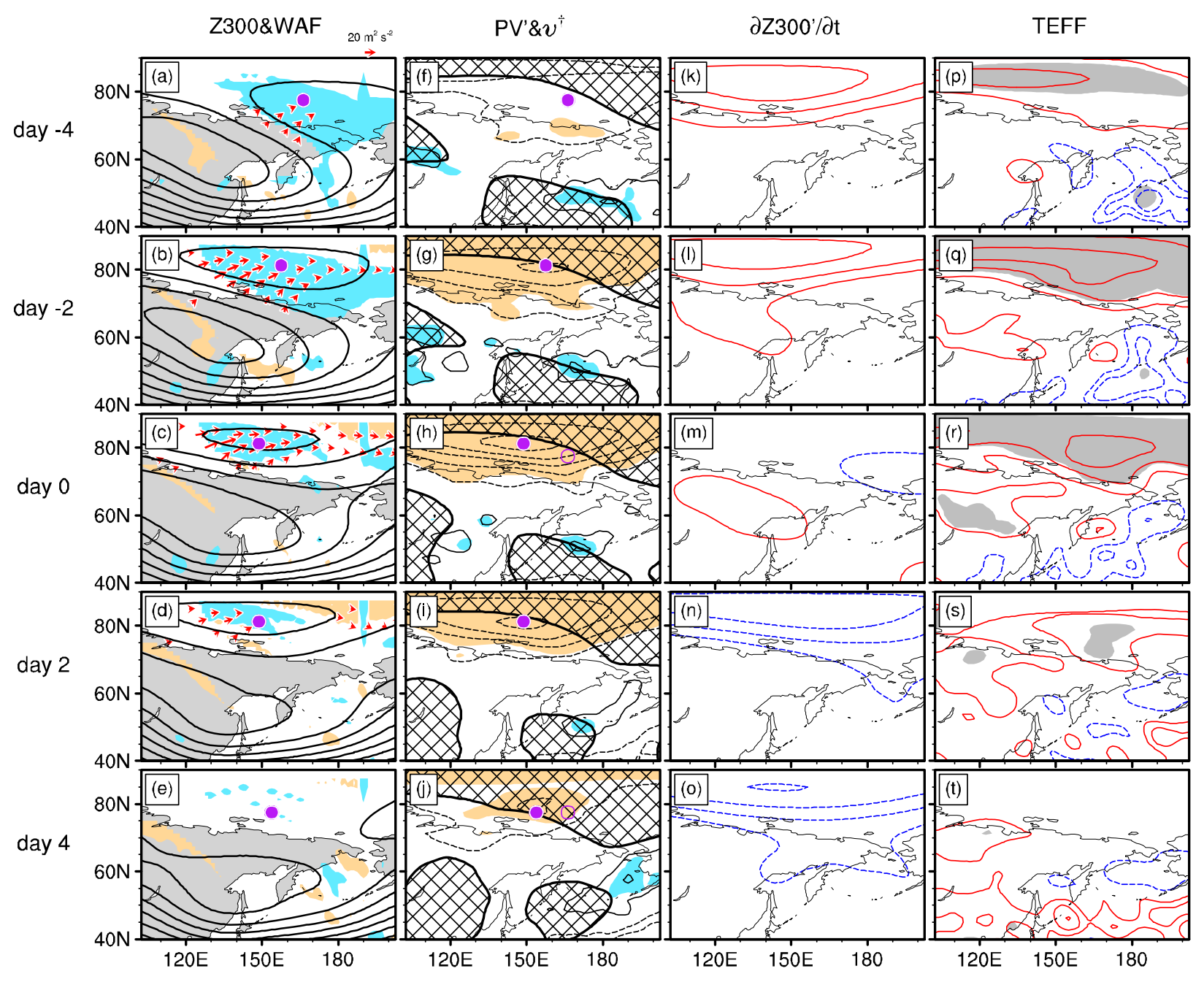

FIG. 7. As in Fig. 5, but for 23 westward-type WBBH events at $83.75^{\circ} \mathrm{N}, 142.5^{\circ} \mathrm{E}$. The southernmost latitude is $40^{\circ} \mathrm{N}$. In (f)-(j), the contour interval is $2 \times 10^{-8} \mathrm{~K} \mathrm{~m}^{2} \mathrm{~kg}^{-1} \mathrm{~s}^{-1}$.

Under both the wave-activity injection and reinforcing TEFF, the primary PV anomaly amplifies at day -2 (Fig. $7 \mathrm{~g}$ ) and then reaches its maximum amplitude at day 0 (Fig. $7 \mathrm{~h})$. At the same time, the blocking high has been detached from the main westerlies over the North Pacific, becoming an isolated anticyclonic vortex over the Arctic Ocean (Figs. 7b-d). As the vortex undergoes rapid decay around day +4 (Fig. 7e), the anomalous PV flux turns into positive and the primary PV anomaly center moves finally into the weak northerlies (Fig. 7j) in a manner consistent with the diminished convergence of the wave-activity flux. However, the flux emanation from the PV anomaly center is negligible, under the extremely weak westerlies around the decaying isolated vortex (Fig. 7e).

In the composited evolution, a westward-type WB feature is evident, especially in the developing stage, as characterized by the northwestward evolution of the primary negative PV anomaly center in association with the rapid development of the ridge into the Arctic Ocean. This evolution may be viewed as cyclonic breaking of the blocking ridge and planetary-wave trough, as typically observed over the northern Far East (Takaya and Nakamura 2005; Rivière et al. 2010; Nishii et al. 2010, 2011).

\section{3) EASTWARD-TYPE WBBL EVENTS}

Figure 8 illustrates composited evolution of 34 WBBL events of the eastward type that develop to the west of Greenland, as a manifestation of the locally enhanced polar vortex around its climatological location (Figs. 8a-c). In the developing stage at days -4 and -2 , the primary positive PV anomaly center is situated in anomalous equatorward winds perpendicular to the climatological-mean flow ( $v^{\dagger}<0$; Figs. 8f,g). The negative anomalous PV flux $\left(v^{\dagger} q^{\prime}<0\right)$ thus yielded is consistent with the wave-activity flux converging into the vicinity of the PV center, especially at day -2 (Figs. 8a,b). The PV anomaly further amplifies until day 0 (Fig. $8 \mathrm{~h}$ ), and then it weakens in moving gradually closer to the southwesterly jet where anomalous wind is poleward $\left(v^{\dagger}>0\right)$ during the decay stage (Figs. 8d,e,i,j). Correspondingly, the wave-activity flux around the positive PV 


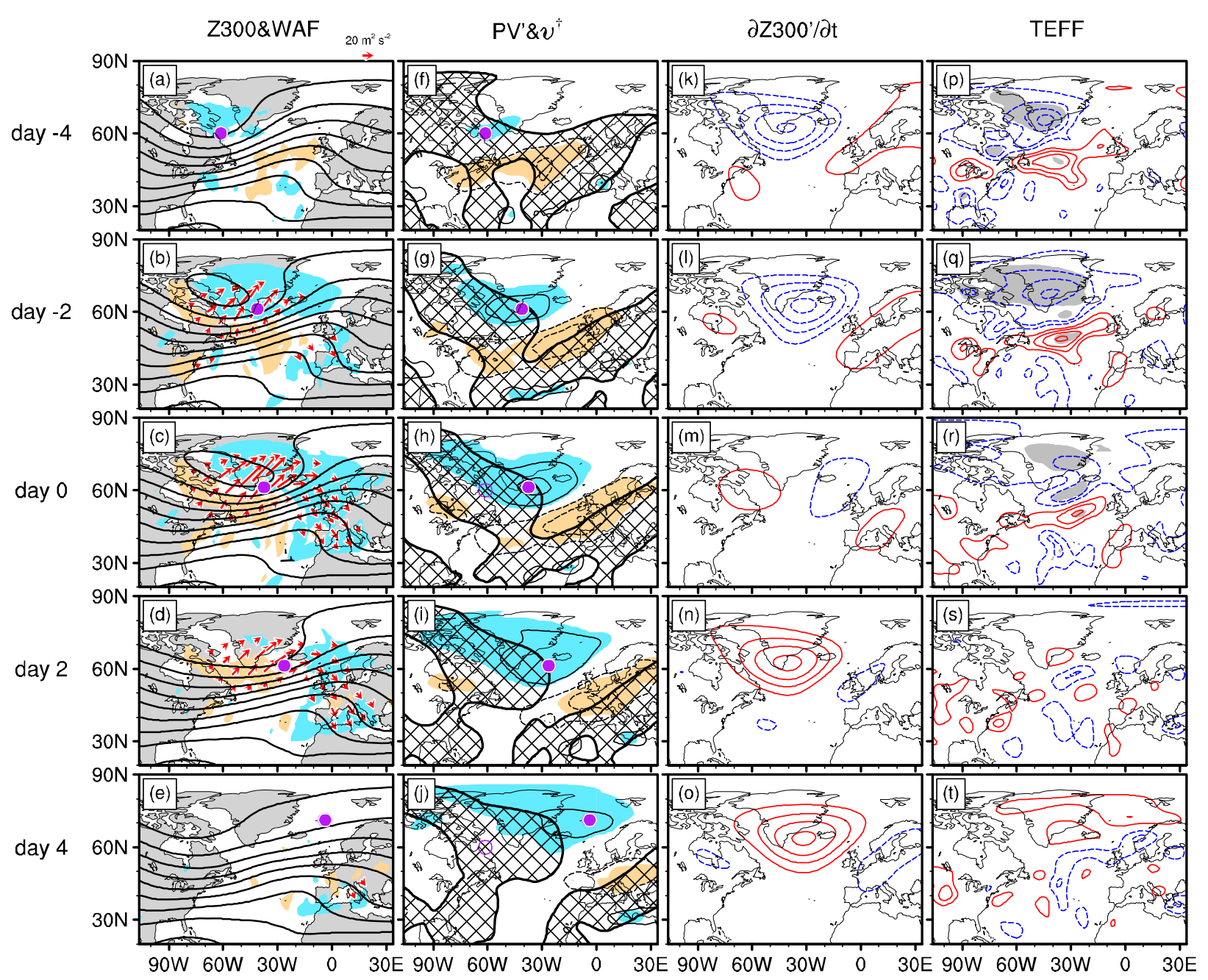

FIG. 8. As in Fig. 5, but for 34 eastward-type WBBL events at $63.75^{\circ} \mathrm{N}, 323.75^{\circ} \mathrm{E}$. Purple dots indicate the center of the primary positive $\mathrm{PV}$ anomaly.

anomalies becomes divergent in the decay stage, especially at day +2 (Fig. 8d).

During the developing stage, TEFF also plays an important role in reinforcing the local deepening of the polar vortex. In fact, it accounts for $\sim 20 \%$ of the anomalous negative height tendency observed around the primary PV anomaly center (Figs. 8k,1,p,q). In the decay stage, the TEFF is weakened and only of marginal significance (Figs. 8s,t).

The eastward-type WB feature associated with the locally enhanced polar vortex is manifested as a gradual eastward movement of the primary positive PV anomaly center across southern Greenland in the developing stage followed by its more rapid northeastward translation in the decaying stage under the cyclonic shear of the Atlantic jet. This WB is concomitant with the development of strong negative PV anomalies from the North Atlantic into Europe, which occurs also under significant positive TEFF (Fig. 8q). The resultant enhancement of the southwesterly jet contributes to the rapid northeastward movement of the primary PV anomaly center
(Figs. 8c,d). In addition, the development of the negative PV anomalies and associated weakening of the westerlies to the south are consistent with the convergence of the southeastward wave-activity flux emanating from the south of the enhanced polar vortex.

\section{4) OTHER TYPES OF WB EVENTS}

The composite evolution of 16 westward-type WBBL events at $75^{\circ} \mathrm{N}, 217.5^{\circ} \mathrm{E}$ features the southwestward movement of the primary PV anomaly center associated with the locally enhanced polar vortex over the Arctic Ocean off Alaska (not shown). The anomalous PV flux is initially negative and then becomes positive after day 0 , corresponding to the waveactivity flux changing from its convergence to its weak divergence around the PV anomaly center. The composited TEFF exerts no significant influence (not shown).

The corresponding composite results for the stationary type of $10 \mathrm{WBBH}$ events at $50^{\circ} \mathrm{N}, 220^{\circ} \mathrm{E}$ and $13 \mathrm{WBBL}$ events at $55^{\circ} \mathrm{N}, 342.5^{\circ} \mathrm{E}$ also show a consistent relationship between the 
anomalous PV flux and propagation of quasi-stationary Rossby waves, but the primary PV anomaly center in the composites seems almost stationary around the peak day (not shown). Due to the small sample size, however, the composited anomalies are marginal significant, especially for TEFF.

\section{Discussion}

Compared with the composite results by Masato et al. (2012), our results for the eastward/westward types show good correspondence to their cyclonic/anticyclonic types. On one hand, the eastward type of WBBH events exhibit a clear anticyclonic overturning feature (our Figs. 5 and 6), while both the westward type of $\mathrm{WBBH}$ events and the eastward type of WBBL events exhibit a cyclonic overturning feature (our Figs. 7 and 8). On the other hand, their anticyclonic type of blocking is characterized by the eastward movement of the positive $\theta_{2 \mathrm{PVU}}$ anomalies and the westward movement of the negative $\theta_{2 \mathrm{PVU}}$ anomalies (their Figs. 5 and 6), while their cyclonic type is characterized by the opposite movements of the $\theta_{2 \mathrm{PVU}}$ anomalies (their Figs. 7-9).

In recognition of the clear connection between eastward/ westward types and previous classifications, a comparison is further made between our results and Masato et al. (2013) on the horizontal frequency distribution of WB/blocking events. The smoother frequency distribution shown by Masato et al. (2013) might be due to the fact that all nearby grid points were incorporated into their frequency statistics. They selected all nearby grid points (around the breaking center) at which the meridional gradient of potential temperature is negative at the tropopause level for all blocking days. In contrast, our method detects only the primary $\mathrm{Z} 300^{\prime}$ centers at the peak day. Furthermore, as pointed out in section 3a, the absolute-field method used by Masato et al. (2013) is likely to lead to fewer blocking events identified over the central Atlantic and the eastern Pacific. In contrast, our results derived from both the absolute-field and anomaly-field methods combined elucidate that both the WBBHs and WBBLs over the two maritime regions are dominated by the eastward type (Fig. 4).

Typically, the breaking center is located equatorward of the primary positive $\mathrm{Z} 300^{\prime}$ center and poleward of the primary negative $\mathrm{Z} 300^{\prime}$ center. Thus, there are some latitudinal displacement and even slight longitudinal displacement between the frequency distribution obtained in our study and by Masato et al. (2013). Specifically, our eastward-type WBBH events are frequent over Scandinavia and the Barents Sea (Fig. 4a), roughly to the north of central Europe, where their "warm-anticyclonic" type is frequently identified (their Fig. 8). Likewise, displacements can also be observed between our westward-type WBBH events over the central Arctic Ocean and eastern Siberia (Fig. 4b) and their "warm-cyclonic" type over the Far East (their Fig. 5), between our eastward-type WBBL events (Fig. 4d) over the Far East and northwestern Pacific and their "cold-cyclonic" type to the farther north (their Fig. 5), and between our westward-type WBBH events over the Baffin Island (Fig. 4b) and their "cyclonic" types around the Davis Strait (their Fig. 8).

Let us go back to the 14-day period in January 2012, as shown in Fig. 1. In fact, our method successfully distinguishes two successive WB events: an eastward-type WBBL event and a stationary WBBH event that peaked on 12 and 19 January, respectively. Not surprisingly, these peak days are basically consistent with those identified by the extreme RI index (Fig. 1a), which is based on departure fields (Masato et al. 2013). Still, our results are on a stronger dynamical basis. On one hand, our result is consistent with the amplification and southeastward development of the planetary-wave trough over the northern Far East and the subsequent development of a blocking ridge to the immediate east that evolved from a retrograding anticyclonic anomaly over the North Pacific (Figs. 1b,c). Thus, these two events consecutively evolved in exhibiting apparently different WB features, and we therefore consider it more appropriate to identify them as separate WB events rather than as a single blocking or WB event. Nevertheless, this result suggests that these WB events may be interpreted as consecutive amplification of the cyclonic and anticyclonic anomalies associated with a quasistationary Rossby wave train propagating northeastward.

In re-examining many other WB events identified through the PV $-\theta$ method, we have found that some of them cannot be identified as our WB events, since they accompany weak or modest anomalies or even lack of local breaking of Rossby wave trains. Meanwhile, we have also found that some of our WB events cannot be identified through the PV $-\theta$ method since they are not characterized by reversed-gradient features (not shown), especially over the regions with relatively strong meridional PV gradient (e.g., the central North Atlantic). These differences by no means suggest which method is better, but rather they arise from which aspects of WB are highlighted in a given method. From this perspective, our method is more suited for detecting WB events occurring with strong circulation anomalies associated with quasistationary Rossby waves.

A numerical study by Orlanski (2003) suggests that intensity of the near-surface baroclinic development of disturbances could influence the orientation of upper-level WB, which could, in turn, influence the jet position, indicating possible interaction between the jet stream and WB morphology. Tyrlis and Hoskins (2008b) reviewed earlier works, including Thorncroft et al. (1993) and Peters and Waugh (1996), to argue that the jet position and ambient shear can control the WB morphology. Abatzoglou and Magnusdottir (2006) found that the seasonal evolution of the background zonal flow can regulate the climatological location and frequency of "anticyclonic" planetary-wave breaking in the subtropics. Motivated by these studies, we include Fig. 9, which shows the local westerly shear composited separately for the three types of WB events on their peak days. As in Nishii et al. (2011), the westerly shear is represented by the difference of the maximum low-pass-filtered westerly wind speed to the north of the $\mathrm{Z}^{3} 00^{\prime}$ center from its counterpart to its south. This method is based on the fact that a blocking flow configuration is generally accompanied by a split-jet configuration. For a WBBH event, its primary $\mathrm{Z} 300^{\prime}$ center is supposed to be situated closer to the northern branch than to the southern branch of the split westerlies, while the opposite is supposed to be the case for a WBBL event. In taking these different flow configurations between WBBHs and WBBLs into account, for a given $\mathrm{WBBH}$ 
eastward-type WBBH

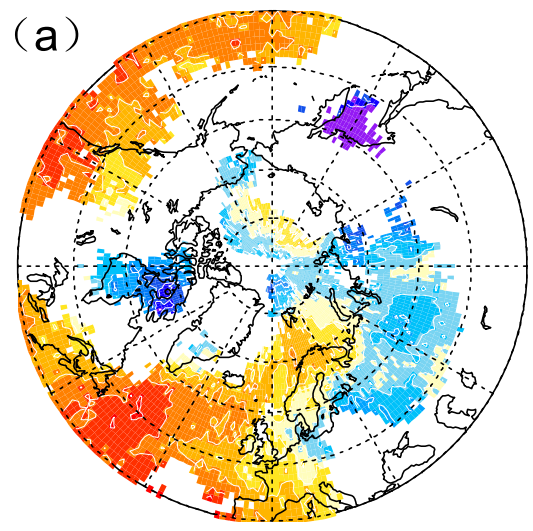

westward-type WBBH

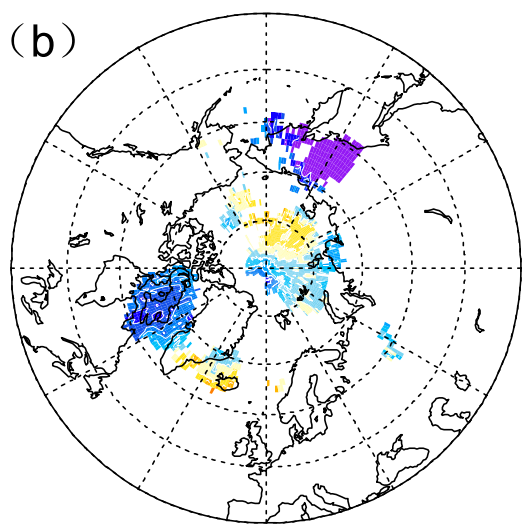

eastward-type WBBL

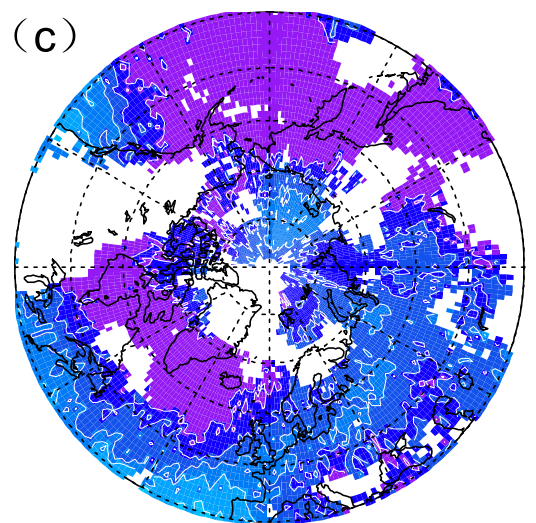

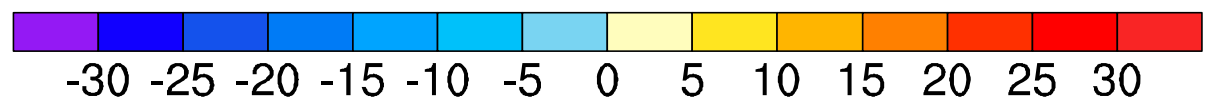

FIG. 9. Maps of composite difference in low-pass-filtered maximum westerly wind speed $\left(\mathrm{m} \mathrm{s}^{-1}\right)$ to the north of the primary Z300' center from that to its south for the (a) eastward-type WBBH, (b) westward-type WBBH, and (c) eastward-type WBBL of high-amplitude WB events with blocking flow configurations at their peak days. The negative and positive differences correspond to cyclonic and anticyclonic westerly shear, respectively, for the WB events and plotted only where at least 10 events are identified for a given type. See more details in the text.

event both the northern and southern maxima of the westerly wind speed are searched within a rectangular domain centered at the grid point $10^{\circ}$ south of the $\mathrm{Z} 300^{\prime}$ center. The rectangular domain is set to $10^{\circ}$ in longitude and $50^{\circ}$ in latitude. The same method is also applied to a WBBL event, but with the search domain centered at $10^{\circ}$ north of the Z300' center. We have confirmed that the results are rather insensitive to slight changes in the size of the search domains. In Fig. 9, the westerly shear thus obtained is plotted only where 10 or more events are identified for a given WB category.

Consistent with Tyrlis and Hoskins (2008b), the eastwardtype WBBH events evolve mainly under anticyclonic westerly shear over the North Atlantic, western Europe, Norwegian Sea, Scandinavia, the central and eastern North Pacific, and western North America (Fig. 9a). Interestingly, the eastwardtype WBBH events evolve also under weak cyclonic shear over central Siberia and strong cyclonic westerly shear over the regions where the planetary-wave troughs are situated climatologically (i.e., Hudson Bay and Sea of Okhotsk; Fig. 9a). Westward-type WBBH events, by contrast, occur primarily under cyclonic shear over eastern Siberia and the Baffin Bay (Fig. 9b). In other words, a WBBH event of either the westward or eastward type can evolve under the cyclonic shear around the planetary-wave troughs. Meanwhile, WBBL events are mostly eastward type and thus occur under cyclonic westerly shear over the midlatitude/subpolar North Pacific, the Atlantic, and the Eurasian continent (Fig. 9c). Our results suggest that background westerly shear alone cannot fully determine which type of a WB event occurs, especially for a WBBH event, although dynamical interpretation of these features requires future investigation.

\section{Conclusions}

The present study has proposed a new scheme for detecting events that exhibit 1) high amplitude, 2) wave-breaking (WB), and 3) blocking flow configurations. Detection starts with identifying high-amplitude events in each of which amplitude of the primary $\mathrm{Z} 300^{\prime}$ center on its peak day exceeds its local unit standard deviation. The most distinct feature of our index from the corresponding conventional methods is the inclusion of the evolution of anomalous PV flux as a dynamical measure of Rossby wave breaking. Only those high-amplitude events that exhibit the sign reversal of the anomalous PV flux at the primary PV anomaly center from negative to positive in time are recognized as the high-amplitude WB events. Finally, a blocking flow configuration is considered to emerge if a local maximum geopotential height at $500 \mathrm{hPa}$ can be found around the primary $\mathrm{Z} 300^{\prime}$ center. Clearly, our method combines the "absolute field" with "departure field" to define a highamplitude WB event with a blocking flow configuration. The polarity of the primary $\mathrm{Z} 300^{\prime}$ center leads to the classification of a given event into a WB blocking high (WBBH) or blocking low (WBBL) event. The longitudinal movement of the primary $\mathrm{PV}$ anomaly center during a given event is used to further classify those WB events into the eastward, westward, or stationary type.

The new algorithm is then applied to the JRA-55 daily data for 57 boreal cold seasons. We have shown that most of the WBBH events are the eastward type, which is most likely to occur under the anticyclonic westerly shear over the North Atlantic and the northeastern Pacific. Although this happens less frequently, eastward-type $\mathrm{WBBH}$ events are found to evolve under the cyclonic westerly shear over Hudson Bay and 
the Sea of Okhotsk near the climatological-mean planetarywave troughs and also over central Siberia. By contrast, WBBHs of the westward type are most likely to develop under the cyclonic westerly shear in the vicinity of the climatologicalmean planetary-wave troughs around the Baffin Bay and eastern Siberia. Meanwhile, almost all the WBBL events are the eastward type evolving under cyclonic westerly shear extensively over the midlatitude and subpolar regions of the Northern Hemisphere. On the whole, the frequency distributions we obtain are essentially consistent with previous studies on WB and blocking events.

Although TEFF generally contributes positively (generally less than $30 \%$ ) to the amplification of the primary height anomaly at the developing stage of WB events, our composite analysis has verified the consistency between a propagating quasi-stationary Rossby wave train and anomalous PV flux. The negative anomalous PV flux generally corresponds to the convergence of the wave-activity flux and the amplification of the high-amplitude WB events, while positive anomalous PV flux corresponds to the diverging wave-activity flux at the decay stage of those events. Interestingly, despite our usage of anomaly fields to identify the evolution of WB events, the composite total fields also exhibit overturning WB features that have been emphasized in the previous studies. Therefore, our new method explicitly incorporates dynamical aspects during the evolution of WB events. The present study presents specific composited flow evolution of high-amplitude WB events with blocking flow configurations only at four representative locations. The corresponding events at different locations and geographical dependence of the WB features, in addition to their seasonality, will be examined in our future study.

Acknowledgments. The authors express sincere thanks to the three anonymous reviewers for their sound criticism and constructive comments that have led to substantial improvement of this paper. Discussion with Dr. Kazuaki Nishii is appreciated. NS is supported jointly by the National Key R\&D Program of China (Grant 2016YFA0600702), the Chinese Natural Science Foundation (41575057, 41975063), the funding of the Jiangsu innovation and entrepreneurship team, and the Qing Lan Project. HN is supported in part by the Japanese Ministry of Environment through the Environment Research and Technology Development Fund 2-1904, by the Japanese Ministry of Education, Culture, Sports, Science and Technology through the ArCS-II Project, by the Japan Society of Promotion of Science through Grants-in-Aid for Scientific Research JP18H01278, JP19H05702 (in Innovative Area 6102) and JP20H01979, and by the Japan Science and Technology Agency through Belmont Forum CRA "InterDec." The NCAR Command Language (NCL) was used for the calculation and drawing the plots.

\section{REFERENCES}

Abatzoglou, J. T., and G. Magnusdottir, 2006: Planetary wave breaking and nonlinear reflection: Seasonal cycle and interannual variability. J. Climate, 19, 6139-6152, https://doi.org/ 10.1175/JCLI3968.1.

Altenhoff, A. M., O. Martius, M. Croci-Maspoli, C. Schwierz, and H. C. Davies, 2008: Linkage of atmospheric blocks and synoptic-scale Rossby waves: A climatological analysis. Tellus, 60A, 1053-1063, https://doi.org/10.1111/j.1600-0870.2008.00354.x. Barriopedro, D., R. García-Herrera, and R. Trigo, 2010: Application of blocking diagnosis methods to general circulation models. Part I: A novel detection scheme. Climate Dyn., 35, 1373-1391, https://doi.org/10.1007/s00382-010-0767-5.

Berrisford, P., B. J. Hoskins, and E. Tyrlis, 2007: Blocking and Rossby wave breaking on the dynamical tropopause in the Southern Hemisphere. J. Atmos. Sci., 64, 2881-2898, https:// doi.org/10.1175/JAS3984.1.

Croci-Maspoli, M., C. Schwierz, and H. C. Davies, 2007: A multifaceted climatology of atmospheric blocking and its recent linear trend. J. Climate, 20, 633-649, https://doi.org/10.1175/ JCLI4029.1.

Diao, Y., J. Li, and D. Luo, 2006: A new blocking index and its application: Blocking action in the Northern Hemisphere. J. Climate, 19, 4819-4839, https://doi.org/10.1175/JCLI3886.1.

Dole, R. M., and N. D. Gordon, 1983: Persistent anomalies of the extratropical Northern Hemisphere wintertime circulation: Geographical distribution and regional persistence characteristics. Mon. Wea. Rev., 111, 1567-1586, https://doi.org/ 10.1175/1520-0493(1983)111<1567:PAOTEN > 2.0.CO;2.

Dunn-Sigouin, E., S.-W. Son, and H. Lin, 2013: Evaluation of Northern Hemisphere blocking climatology in the global environment multiscale model. Mon. Wea. Rev., 141, 707-727, https://doi.org/10.1175/MWR-D-12-00134.1.

Ertel, H., 1942: Ein neuer hydrodynamischer Wirbelsatz. Meteor. Z. Braunchweigs, 59, 271-281.

Holopainen, E., and C. Fortelius, 1987: High-frequency transient eddies and blocking. J. Atmos. Sci., 44, 1632-1645, https://doi.org/ 10.1175/1520-0469(1987)044<1632:HFTEAB > 2.0.CO;2.

Hoskins, B. J., M. E. McIntyre, and A. W. Robertson, 1985: On the use and significance of isentropic potential vorticity maps. Quart. J. Roy. Meteor. Soc., 111, 877-946, https://doi.org/ 10.1002/qj.49711147002.

Huang, C. S. Y., and N. Nakamura, 2016: Local finite-amplitude wave activity as a diagnostic of anomalous weather events. J. Atmos. Sci., 73, 211-229, https://doi.org/10.1175/JAS-D-150194.1.

Kobayashi, S., and Coauthors, 2015: The JRA-55 reanalysis: General specifications and basic characteristics. J. Meteor. Soc. Japan, 93, 5-48, https://doi.org/10.2151/jmsj.2015-001.

Lau, N.-C., 1988: Variability of the observed midlatitude storm tracks in relation to low-frequency changes in the circulation pattern. J. Atmos. Sci., 45, 2718-2743, https://doi.org/10.1175/ 1520-0469(1988)045<2718:VOTOMS>2.0.CO;2.

time-mean flow as identified by geopotential tendencies. J. Atmos. Sci., 41, 313-328, https://doi.org/10.1175/15200469(1984)041<0313:TEFOTT>2.0.CO;2.

_ , and M. J. Nath, 1991: Variability of the baroclinic and barotropic transient eddy forcing associated with monthly changes in the midlatitude storm tracks. J. Atmos. Sci., 48, 2589-2613, https:// doi.org/10.1175/1520-0469(1991)048<2589:VOTBAB >2.0.CO;2.

Lejenäs, H., and H. Økland, 1983: Characteristics of Northern Hemisphere blocking as determined from a long series of observational data. Tellus, 35A, 350-362, https://doi.org/ 10.1111/j.1600-0870.1983.tb00210.x.

Martius, O., C. Schwierz, and H. C. Davies, 2007: Breaking waves at the tropopause in the wintertime Northern Hemisphere: Climatological analyses of the orientation and the theoretical LC1/2 classification. J. Atmos. Sci., 64, 2576-2592, https:// doi.org/10.1175/JAS3977.1. 
Masato, G., B. J. Hoskins, and T. J. Woollings, 2012: Wavebreaking characteristics of midlatitude blocking. Quart. J. Roy. Meteor. Soc., 138, 1285-1296, https://doi.org/10.1002/ qj. 990 .

- — , and — 2013: Wave-breaking characteristics of Northern Hemisphere winter blocking: A two-dimensional approach. J. Climate, 26, 4535-4549, https://doi.org/10.1175/ JCLI-D-12-00240.1.

Nakamura, H., 1994: Rotational evolution of potential vorticity associated with a strong blocking flow configuration over Europe. Geophys. Res. Lett., 21, 2003-2006, https://doi.org/ 10.1029/94GL01614.

— , and T. Fukamachi, 2004: Evolution and dynamics of summertime blocking over the Far East and the associated surface Okhotsk high. Quart. J. Roy. Meteor. Soc., 130, 1213-1233, https://doi.org/10.1256/qj.03.101.

—_, M. Nakamura, and J. L. Anderson, 1997: The role of highand low-frequency dynamics in blocking formation. Mon. Wea. Rev., 125, 2074-2093, https://doi.org/10.1175/15200493(1997)125<2074:TROHAL $>2.0$.CO;2.

Nishii, K., T. Miyasaka, and H. Nakamura, 2009: Modulations in the planetary wave field induced by upward-propagating Rossby wave packets prior to stratospheric sudden warming events: A case study. Quart. J. Roy. Meteor. Soc., 135, 39-52, https://doi.org/10.1002/qj.359.

- , H. Nakamura, and Y. J. Orsolini, 2010: Cooling of the wintertime Arctic stratosphere induced by the western Pacific teleconnection pattern. Geophys. Res. Lett., 37, L13805, https://doi.org/10.1029/2010GL043551.

,$--\frac{1}{-}$, and -2011 : Geographical dependence observed in blocking high influence on the stratospheric variability through enhancement and suppression of upward planetarywave propagation. J. Climate, 24, 6408-6423, https://doi.org/ 10.1175/JCLI-D-10-05021.1.

Orlanski, I., 2003: Bifurcation in eddy life cycles: Implications for storm track variability. J. Atmos. Sci., 60, 993-1023, https:// doi.org/10.1175/1520-0469(2003)60<993:BIELCI >2.0.CO;2.

Pelly, J. L., and B. J. Hoskins, 2003: A new perspective on blocking. J. Atmos. Sci., 60, 743-755, https://doi.org/10.1175/ 1520-0469(2003)060<0743:ANPOB >2.0.CO;2.

Peters, D., and D. W. Waugh, 1996: Influence of barotropic shear on the poleward advection of upper-tropospheric air. J. Atmos. Sci., 53, 3013-3031, https://doi.org/10.1175/15200469(1996)053<3013:IOBSOT>2.0.CO;2.

Pinheiro, M. C., P. A. Ullrich, and R. Grotjahn, 2019: Atmospheric blocking and intercomparison of objective detection methods: Flow field characteristics. Climate Dyn., 53, 4189-4216, https:// doi.org/10.1007/s00382-019-04782-5.

Rex, D. F., 1950: Blocking action in the middle troposphere and its effect upon regional climate. Tellus, 2, 196-211, https:// doi.org/10.1111/j.2153-3490.1950.tb00331.x.

Rivière, G., A. Laîné, G. Lapeyre, D. Salas-Mélia, and M. Kageyama, 2010: Links between Rossby wave breaking and the North Atlantic Oscillation-Arctic Oscillation in present-day and Last Glacial Maximum climate simulations. J. Climate, 23, 2987-3008, https://doi.org/10.1175/2010JCLI3372.1.

Rossby, C. G., 1940: Planetary flow patterns in the atmosphere. Quart. J. Roy. Meteor. Soc., 66, 68-87.

Schwierz, C., M. Croci-Maspoli, and H. C. Davies, 2004: Perspicacious indicators of atmospheric blocking. Geophys. Res. Lett., 31, L06125, https://doi.org/10.1029/2003GL 019341.
Shukla, J., and K. C. Mo, 1983: Seasonal and geographical variation of blocking. Mon. Wea. Rev., 111, 388-402, https://doi.org/ 10.1175/1520-0493(1983)111<0388:SAGVOB>2.0.CO;2.

Small, D., E. Atallah, and J. R. Gyakum, 2014: An objectively determined blocking index and its Northern Hemisphere climatology. J. Climate, 27, 2948-2970, https://doi.org/10.1175/ JCLI-D-13-00374.1.

Strong, C., and G. Magnusdottir, 2008: Tropospheric Rossby wave breaking and the NAO/NAM. J. Atmos. Sci., 65, 2861-2876, https://doi.org/10.1175/2008jas2632.1.

Takaya, K., and H. Nakamura, 2001: A formulation of a phaseindependent wave-activity flux for stationary and migratory quasigeostrophic eddies on a zonally varying basic flow. J. Atmos. Sci., 58, 608-627, https://doi.org/10.1175/15200469(2001)058<0608:AFOAPI > 2.0.CO;2.

$\longrightarrow$, and — 2005: Geographical dependence of upper-level blocking formation associated with intraseasonal amplification of the Siberian high. J. Atmos. Sci., 62, 4441-4449, https:// doi.org/10.1175/JAS3628.1.

Thorncroft, C. D., B. J. Hoskins, and M. E. McIntyre, 1993: Two paradigms of baroclinic-wave life-cycle behaviour. Quart. J. Roy. Meteor. Soc., 119, 17-55, https://doi.org/10.1002/ qj.49711950903.

Tibaldi, S., and F. Molteni, 1990: On the operational predictability of blocking. Tellus, 42A, 343-365, https://doi.org/10.3402/ tellusa.v42i3.11882.

Trenberth, K. E., 1986: An assessment of the impact of transient eddies on the zonal flow during a blocking episode using localized Eliassen-Palm flux diagnostics. J. Atmos. Sci., 43, 2070-2087, https://doi.org/10.1175/1520-0469(1986)043<2070: AAOTIO $>2.0 . C O ; 2$.

Tyrlis, E., and B. J. Hoskins, 2008a: Aspects of a Northern Hemisphere atmospheric blocking climatology. J. Atmos. Sci., 65, 1638-1652, https://doi.org/10.1175/2007JAS2337.1.

- and - 2008b: The morphology of Northern Hemisphere blocking. J. Atmos. Sci., 65, 1653-1665, https://doi.org/10.1175/ 2007JAS2338.1.

Weijenborg, C., H. Vries, and R. J. Haarsma, 2012: On the direction of Rossby wave breaking in blocking. Climate Dyn., 39, 2823-2831, https://doi.org/10.1007/s00382-012-1332-1.

Wilks, D. S., 2016: “The stippling shows statistically significant grid points": How research results are routinely overstated and overinterpreted, and what to do about it. Bull. Amer. Meteor. Soc., 97, 2263-2273, https://doi.org/10.1175/BAMSD-15-00267.1.

Wolf, G., and V. Wirth, 2017: Diagnosing the horizontal propagation of Rossby wave packets along the midlatitude waveguide. Mon. Wea. Rev., 145, 3247-3264, https://doi.org/10.1175/ MWR-D-16-0355.1.

— D. J. Brayshaw, N. P. Klingaman, and A. Czaja, 2018: Quasistationary waves and their impact on European weather and extreme events. Quart. J. Roy. Meteor. Soc., 144, 2431-2448, https://doi.org/10.1002/qj.3310.

Woollings, T., B. Hoskins, M. Blackburn, and P. Berrisford, 2008: A new Rossby wave-breaking interpretation of the North Atlantic Oscillation. J. Atmos. Sci., 65, 609-626, https:// doi.org/10.1175/2007JAS2347.1.

Zschenderlein, P., G. Fragkoulidis, A. H. Fink, and V. Wirth, 2018: Large-scale Rossby wave and synoptic-scale dynamic analyses of the unusually late 2016 heatwave over Europe. Weather, 73, 275-283, https://doi.org/10.1002/wea.3278. 